\title{
Layer Construction of 3D Topological States and String Braiding Statistics
}

\author{
Chao-Ming Jian and Xiao-Liang Qi \\ Department of Physics, Stanford University, Stanford, California 94305, USA
}

(Received 28 June 2014; published 10 December 2014)

\begin{abstract}
While the topological order in two dimensions has been studied extensively since the discovery of the integer and fractional quantum Hall systems, topological states in three spatial dimensions are much less understood. In this paper, we propose a general formalism for constructing a large class of threedimensional topological states by stacking layers of 2D topological states and introducing coupling between them. Using this construction, different types of topological states can be obtained, including those with only surface topological order and no bulk topological quasiparticles, and those with topological order both in the bulk and at the surface. For both classes of states, we study its generic properties and present several explicit examples. As an interesting consequence of this construction, we obtain example systems with nontrivial braiding statistics between string excitations. In addition to studying the string-string braiding in the example system, we propose a topological field-theory description for the layer-constructed systems, which captures not only the string-particle braiding statistics but also the string-string braiding statistics when the coupling is twisted. Last, we provide a proof of a general identity for Abelian string statistics and discuss an example system with non-Abelian strings.
\end{abstract}

DOI: $10.1103 / P h y s R e v X .4 .041043$

\section{INTRODUCTION}

Some of the most important discoveries in condensedmatter physics over the last few decades have been about topological states of matter. Topological states are gapped states of matter that are not characterized by broken symmetries and local order parameters but are still distinct phases from a trivial insulator. A subclass of topological states of matter is the topologically ordered states, which are stable against any local perturbations and have topologically protected properties, including fractional quasiparticle statistics, ground-state degeneracy determined by topology of the spatial manifold, etc. [1]. Since the discovery of integer and fractional quantum Hall effects [2,3], 2D topologically ordered states have been characterized and constructed by many different approaches, such as ideal wave functions [4-7], topological field theories [8,9], exact lattice models [10-13], and tensor networks [14-19]. The general structure of topological states in 2D is governed by the mathematical framework of tensorcategory theory $[12,20,21]$. Since the discovery of topological insulators [22,23], new types of topologically ordered states with nontrivial symmetry properties have also been studied, known as symmetry-protected

Published by the American Physical Society under the terms of the Creative Commons Attribution 3.0 License. Further distribution of this work must maintain attribution to the author(s) and the published article's title, journal citation, and DOI.
Subject Areas: Condensed Matter Physics, Strongly Correlated Materials topological (SPT) states and symmetry-enriched topological states [24-27].

In contrast to the progress in $2 \mathrm{D}$, much less is known about topologically ordered states in $3 \mathrm{D}$. Since there is not yet a general framework for 3D topologically ordered states, building different models with topological order is an important method that can help us understand the general features of 3D topological order. The 3D topological insulators $[22,23]$ can be generalized to fractional topological insulators with topological order and fractionally charged partons [28-31]. Some exactly solvable models in $2 \mathrm{D}$ can be generalized to 3D. For example, the $3 \mathrm{D}$ generalization of the toric code model can capture the topological order of 3D lattice discrete gauge theory in which the ground-state degeneracy is associated with the nontrivial one-cycles and the point particles have nontrivial mutual braiding statistics with flux-string excitations. The Levin-Wen models can be generalized to the Walker-Wang models [32,33] that describe 3D gapped states with interesting surface topological order and 3D bulk topological order that generalizes the 3D lattice gauge theory. In $3 \mathrm{D}$, the statistics between particles is a representation of the permutation group instead of the braid group in 2D, so that there cannot be fractional statistics for pointlike particles $[34,35]$ (although nontrivial statistics have been shown to be possible for pointlike monopole defects [36-39]). However, 3D topologically ordered states can have 1D stringlike excitations in addition to particles. The existence of string excitation enables rich possibilities of braiding statistics. Recently, the topological properties of the lattice 
models of the $3+1 D$ Dijkgraaf-Witten lattice gauge theory [40-43] and 3D generalization of quantum double models $[44,45]$ were studied. In these models, string excitations have nontrivial fusion and braiding statistics.

In this paper, we propose a different approach of constructing 3D topological states named the layer construction. In this construction, 3D topological states can emerge in a system with stacked layers of 2D topological states and properly engineered interlayer couplings. This type of construction is first proposed in a special example of 3D boson topological insulators in Ref. [46]. It is reminiscent of the wire construction of 2D (intrinsic and symmetry-protected) topological states [47-50]. It is shown that if we take an array of the 1D wires of Luttinger liquids and engineer the interwire coupling, this array of wires can effectively form a 2D quantum Hall liquid. Depending on the coupling, a large class of fractional quantum Hall states can be constructed. Motivated by the success of the wire construction for 2D topological states and by the example in Ref. [46], we study in this paper the layer construction of 3D states as a more general formalism of constructing and characterizing 3D topological states. In this general formalism, we consider coupling layers of 2D Abelian topological states where the consequence of the coupling is to induce anyon condensation of some composite particle formed by topological quasiparticles in two adjacent layers. By several explicit examples, we show that this approach can describe a large class of different 3D topological states, including those with only surface 2D topological order and those with bulk and/or surface topological order. For the systems with only surface topological order and no bulk topological order, we construct a series of examples that are $3 \mathrm{D}$ gapped states with interesting chiral 2D topological order on their surfaces, although each layer of 2D states in the construction is nonchiral. This construction of chiral surface state is similar to the observation of Refs. [33,51] in Walker-Wang models. We obtain general criteria for the absence of 3D topological order and the characterization of surface topological order. With these criteria satisfied, we show how to determine the surface topological order on the top, bottom, and side surfaces in a general situation and show the equivalence of different surfaces, which proves that the states obtained in this construction are topologically isotropic. For more general states with nontrivial 3D topological order, our layer construction enables description of the bulk point particles and stringlike excitations. We provide several examples of different topological orders, including a "conventional" 3D topological order that resembles the lattice gauge theory, and more general systems with coexisting bulk and surface topological order. As a particularly interesting feature of this construction, we can construct some 3D topologically ordered states with nontrivial string-string braiding statistics, in addition to the usual particle-string braiding statistics. For the layerconstructed models, we provide a twisted topological field-theory description of the 3D topological order and the string-string and particle-string braiding statistics. We also discuss the general features of string braiding and provide a more general proof of a constraint on string braiding statistics proposed recently by Ref. [41]. In the end, we give an example system with non-Abelian string braiding statistics, to show that more general topological orders are possible in 3D.

The rest of the paper is organized as follows. In Sec. II, we propose a general framework for the layer construction with layers of 2D Abelian topological states. We introduce the coupling between layers by turning on anyon condensations [52-55] for certain composite quasiparticles living in two adjacent layers. We provide general requirements on such condensed particles. In Sec. III, we focus on the construction of 3D gapped states with only 2D topological order on their surfaces and no bulk topological order. Section III A presents several examples, and Sec. III B discusses the general criteria of surface-only topological order. Section III C studies the surface topological order on the top, bottom, and side surfaces and proves their equivalence. In Sec. IV, we discuss more general states with bulk topological order. Section IVA discusses the conventional topological order of the type of discrete gauge theories. Section IV B discusses examples with bulk topological order coexisting with surface topological order. Section IV C discusses the most nontrivial types of system with nontrivial string-string braiding statistics. In Sec. V, we provide a field-theory description of the topological order and the string braiding statistics for the layer-constructed models. Section VI is devoted to more general discussions on string braiding statistics. In Sec. VI A, we prove a general identity that should be satisfied by Abelian string braiding statistics. In Sec. VIB, we we provide an example of non-Abelian strings and their statistics. Section VII concludes the paper with some discussions on open questions and future directions.

\section{GENERAL SETTING OF THE LAYER CONSTRUCTION}

In this section, we will introduce the general formalism for the construction of 3D topological states from layers of 2D topological states. In general, one can start with any topological state in each 2D layer. For concreteness of the discussion, we restrict ourselves to only consider layers of 2D Abelian topological states. Before we introduce the general framework of the layer construction, we will first briefly review the theory of 2D Abelian topological states and the condensation of certain quasiparticles in these states. 2D Abelian topological states can be described by the Abelian Chern-Simons theory with a $K$ matrix, of which the Lagrangian [56] is given by

$$
L_{\mathrm{CS}}=\int d x d y d t \frac{1}{4 \pi} \epsilon^{\mu \nu \lambda} K_{I J} a_{\mu}^{I} \partial_{\nu} a_{\lambda}^{J},
$$


where $a^{I}$ for $I=1, \ldots, r$ are compact $U(1)$ gauge fields and $K$ is a nonsingular integer-symmetric matrix. Here, we have denoted the dimension of the $K$ matrix as $r=\operatorname{dim}(K)$. The quasiparticles in this theory are labeled by $r$-component integer-valued vectors. The topological spin of the quasiparticle labeled by the integer vector $l$ is given by $\theta_{l}=\pi l^{T} K^{-1} l$, and the mutual statistics of two quasiparticles $l$ and $l^{\prime}$ is $\theta_{l l^{\prime}}=2 \pi l^{T} K^{-1} l^{\prime}$. A particle labeled by $l=K v$, with $v$ an $r$-component integer vector, is considered as a local particle in the theory. In this theory, we can further consider the condensation of a subgroup of the quasiparticles $M_{\text {Lag }}$, called a "Lagrangian subgroup," which has the following properties [52-54]. 1. $e^{i \theta_{m}}=e^{i \theta_{m m^{\prime}}}=1$, for all $m, m^{\prime} \in M_{\mathrm{Lag}}$, and 2. for all $l \notin M_{\mathrm{Lag}}, e^{i \theta_{l m}} \neq 1$ for at least one $m \in M_{\text {Lag }}$. The condensation of a Lagrangian subgroup drives a transition from the Abelian topological state to a topologically trivial phase. For each Lagrangian subgroup, there is an equivalent description of the condensate using another set of particles $M_{\text {null }}$ [52-54]. We refer to the particles in this set as null particles. This set $M_{\text {null }}$ is closed under particle fusion and satisfies the following conditions. 1. $\theta_{m}=\theta_{m m^{\prime}}=0$, for all $m, m^{\prime} \in M_{\text {null }}$, and 2. $M_{\text {null }}$ is generated by $\operatorname{rank}\left(M_{\text {null }}\right)=r / 2$ null particles $m_{i}$, $i=1, \ldots, r / 2$. Notice that for the Lagrangian subgroup or the null-particle set to exist, the dimension of the $K$ matrix $r$ has to be even. It has been proven that the Lagrangian subgroups $M_{\text {Lag }}$ and the null-particle sets $M_{\text {null }}$ are in one-toone correspondence to each other [57]. The condensation of the particles of the null set $M_{\text {null }}$ will induce the condensation of all the particles in its corresponding Lagrangian subgroup $M_{\text {Lag }}$ modulo the local particles that can be always thought of as condensed, and vice versa.

Now, we can start introducing the general setting of the layer construction of the 3D topological states. First, we consider stacked layers of the identical 2D Abelian topological states with the $K$ matrix $K$ for each layer. Before we introduce the coupling between the layers, we can view the system as a 2D state using the $2+1 \mathrm{D}$ Chern-Simons theory with an extended $K$ matrix $\mathcal{K}$ given by

$$
\mathcal{K}=K \otimes\left(\begin{array}{lll}
1 & & \\
& 1 & \\
& & \ddots
\end{array}\right)_{L \times L},
$$

where $L$ stands for the number of layers in the system. We can turn on the "local" coupling between the layers by introducing the condensation of composite quasiparticles that lives in a finite range of layers. Without the loss of generality, we only consider the composite particles that live in two consecutive layers, as is shown in Fig. 1(a). These particles, which we label as $n_{i}^{(m)}$, take the form of

$$
n_{i}^{(m)}=p_{i} \otimes e_{m}+q_{i} \otimes e_{m+1},
$$

(a)

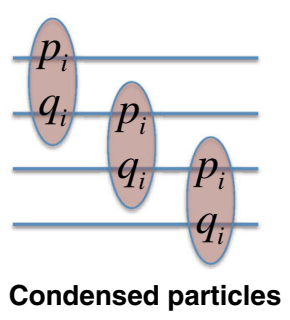

(b)

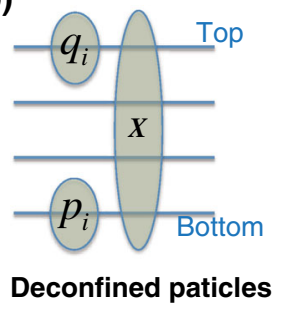

FIG. 1. (a) Each line represents a layer of a 2D Abelian topological state, and each brown circle represents a composite particle, the condensation of which will introduce the coupling between layers. Here, each composite particle lives only in two consecutive layers, and the component of the particle in the upper and lower layers is given by $p_{i}$ 's and $q_{i}$, respectively. (b) For an open boundary system in the $z$ direction, $q_{i}$ 's $\left(p_{i}\right.$ 's) are deconfined particles on the top (bottom) surface that will form surface topological order. Depending on the choice of $\left\{p_{i}\right\}$ and $\left\{q_{i}\right\}$, there can be deconfined particles (represented by " $X$ ") in the $3 \mathrm{D}$ bulk that organize themselves to form 3D topological orders.

where $p_{i}$ 's and $q_{i}$ 's are $r$-component integer vectors that label the quasiparticle type in one single layer and $e_{m}$ is an $L$-component unit vector with the $m$ th entry 1 and all the rest 0 . The superscript $m$ is effectively the layer index, which will later be identified as the "lattice coordinate" in the $z$ direction. For a system that is periodic in the perpendicular $z$ direction, we need to consider $m=$ $1,2, \ldots, L$ with the identification that $e_{L+1}=e_{1}$ while, for the open boundary condition in the $z$ direction, we only need to consider the condensation of null particles with $m=1,2, \ldots, L-1$. The subscript $i$ labels the different types of the condensed quasiparticles $n_{i}^{(m)}$. It should be noticed that the condensed particles are completely determined by the sets $\left\{p_{i}\right\}$ and $\left\{q_{i}\right\}$ that are independent from the layer index $m$. Thus, the condensate of these particles is invariant under the cyclic permutation between the layer, namely, under $m \rightarrow m+1$, and therefore is translationally invariant in the $z$ direction. For the sets $\left\{p_{i}\right\}$ and $\left\{q_{i}\right\}$, we require them to satisfy the following null conditions:

$$
\begin{aligned}
& p_{i}^{T} K^{-1} p_{j}+q_{i}^{T} K^{-1} q_{j}=0, \\
& p_{i}^{T} K^{-1} q_{j}=0, \quad \forall i \text { and } j,
\end{aligned}
$$

such that the mutual statistics and the topological spins of the condensed particles $n_{i}^{(m)}$ are guaranteed to be trivial:

$$
n_{i}^{(m) T} \mathcal{K}^{-1} n_{i^{\prime}}^{\left(m^{\prime}\right)}=0, \quad \forall i, i^{\prime}, m, m^{\prime} .
$$

This condition is similar to the null conditions of the nullparticle set $M_{\text {null }}$ for the 2D Abelian topological state introduced above. Moreover, in analogy to the requirement to the rank of the null set $\operatorname{rank}\left(M_{\text {null }}\right)$, here, we also require that

the number of $p_{i}$ 's $=$ the number of $q_{i}$ 's $=\operatorname{dim}(K) / 2=r / 2$. 
That is to say, the index $i$ takes the values $i=1,2, \ldots, r / 2$. In this counting requirement, we have implicitly assumed that for a given $m$, the choice of $\left\{p_{i}\right\}$ and $\left\{q_{i}\right\}$ guarantees that all particles $n_{i}^{m}$ for $i=1, \ldots, r / 2$ are linearly independent. Again, we only consider cases with even $r$. Here, the choice of the sets $\left\{p_{i}\right\}$ and $\left\{q_{i}\right\}$ is subject to an equivalence relation. For any integer matrix $W$ with $\operatorname{det}(W)=1$, we can define new sets of $\left\{p_{i}^{\prime}\right\}$ and $\left\{q_{i}^{\prime}\right\}$ by the linear transformation $p_{j}^{\prime}=\sum_{i} W_{i j} p_{i}$ and $q_{j}^{\prime}=$ $\sum_{i} W_{i j} q_{i}$ so that the condensed particle $\left\{{n^{\prime}}_{i}^{(m)}\right\}$ defined by the new sets satisfies ${n_{j}^{\prime}}_{j}^{(m)}=\sum_{i} W_{i j} n_{i}^{(m)}$. It is obvious that the condensation of $\left\{n_{i}^{(m)}\right\}$ is equivalent to that of $\left\{n_{i}^{(m)}\right\}$. Therefore, the choice of sets $\left\{p_{i}\right\}$ and $\left\{q_{i}\right\}$ is defined modular a linear transformation by an integer unideterminant matrix $W$.

To summarize the general framework of this construction, we start with layers of 2D Abelian topological states with each layer described by a $K$-matrix theory. Then, in order to couple the layer together to form a 3D topological state, we turn on the condensation of the particles of the form given in Eq. (3), which is determined by the sets $\left\{p_{i}\right\}$ and $\left\{q_{i}\right\}$ that satisfy the null conditions in Eq. (4) and the counting requirements in Eq. (6). Before we move on to more detailed analysis of the layer construction, a couple of comments are in order. First, we argue that the layer construction indeed generates a genuine 3D state. The reasons are the following. (1) It is shown in Refs. [52-54] that the condensation of the null particles can be induced by introducing coupling using only local variables. Here, the locality is understood in the $2 \mathrm{D}$ sense. (2) We only condense composite particles that live in two consecutive layers. Therefore, the locality in the $z$ direction is also guaranteed. (3) The condensation of each quasiparticle is a phase transition from one gapped state to another gapped state. Therefore, the resulting states of the layer construction should also be a gapped 3D state. (4) The requirements [Eqs. (4) and (6)] on the defining data $K,\left\{p_{i}\right\}$ and $\left\{q_{i}\right\}$ do not depend on the number of layers $L$, and the number of condensed null particles $n_{i}^{m}$ is always equal to $\operatorname{dim}(\mathcal{K}) / 2$. The 3D states constructed this way are stable in the thermodynamical limit, and the remaining degrees of freedom under the condensation will not increase as $L \rightarrow \infty$. Second, these layer-constructed 3D states do have topologically nontrivial properties. It is straightforward to see [from Eq. (4)] that, for the open boundary condition, the particle set $\left\{q_{i}\right\}\left(\left\{p_{i}\right\}\right)$ is deconfined on the top (bottom) surface, as is shown in Fig. 1(b). Such 3D states can host 2D topological order on their surfaces, which is similar to the Walker-Wang model with a modular tensor category $[32,33]$. Later, we will see that depending on the choices of $\left\{p_{i}\right\}$ and $\left\{q_{i}\right\}$, we can also have deconfined particles in the $3 \mathrm{D}$ bulk that organize themselves to form 3D topological orders. In the following sections, we will introduce rigorous results and examples of 3D topological states using the layer construction.

As is mentioned above, this discussion on the general setting of the layer construction is partly motivated by a specific example in Ref. [46] in which a specific layerconstructed system was proposed to model the 3D bosonic topological insulator with time-reversal-invariant gapped surface states. Instead of focusing on a specific model, our discussion here mainly emphasizes providing the general setting for the layer construction (at least for Abelian layers) through which one can study various aspects of 3D topological order and 2D topological order on the surface of 3D gapped states. In our general layer construction so far, symmetry is not yet considered but can certainly be incorporated. However, the discussion on layer construction under some certain symmetry is beyond the scope of this paper and will be left for future works.

\section{3D STATES WITH PURELY SURFACE TOPOLOGICAL ORDER}

In this section, we will first introduce a series of examples of 3D states with purely surface topological order (and no bulk topological order). Then, we will discuss the general criteria for the construction of 3D states with purely surface topological orders and a Hamiltonian formalism for their surface topological orders.

\section{A. Example: Coupled layers of the $Z_{p}$ toric code with nontrivial surface topological order}

Reference [46] provides a layer-constructed model of a 3D bosonic topological insulator with time-reversalinvariant gapped surface states. Motivated by this example, we study the generalization of it and obtain a series of layerconstructed models with interesting topologically ordered surfaces. Let us consider the stacked layers of $Z_{p}$ toric code theory. Each layer of $Z_{p}$ toric code theory can be described by the $K$-matrix theory $[10,11,58]$ with

$$
K_{Z_{p}}=\left(\begin{array}{ll}
0 & p \\
p & 0
\end{array}\right) .
$$

There are two types of elementary quasiparticles in the $Z_{p}$ toric code: the electric particle $(1,0)^{T}$ (which will also be labeled as the $e$ particles) and the magnetic particle $(0,1)^{T}$ (which will also be labeled as the $m$ particles). In the stacked-layer theory, the null particles we choose to condense take the following form:

$$
(1,1)^{T} \otimes e_{n}+(1,0)^{T} \otimes e_{n+1}+(1,-1)^{T} \otimes e_{n+2},
$$

which is the composite particle of $e+m, e$, and $e-m$ in three consecutive layers, as is shown in Fig. 2(a). Although we consider composite particles that live in three consecutive layers, we can still recast this type of theory back to the 
(a)

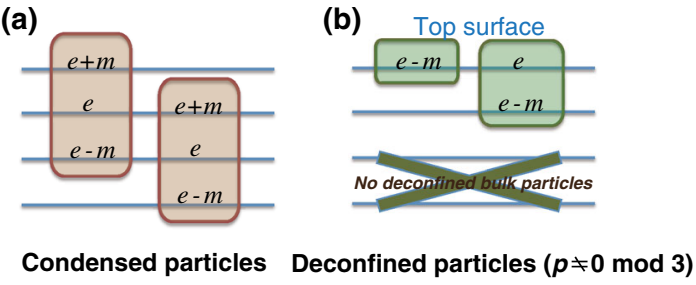

FIG. 2. (a) Illustration of the condensation of the composite particle of $e+m, e$, and $e-m$ in three consecutive layers, with each layer a $Z_{p}$ toric code state. (b) For $p \equiv 1,2 \bmod 3$, there is no deconfined particle in the $3 \mathrm{D}$ bulk. The deconfined particles only stay on the surface, which is generated by two elementary particles shown in the green regions. (The case of $p \equiv 0 \bmod 3$ is discussed later in Fig. 7.)

general setting described in Sec. II by viewing two layers of $Z_{p}$ toric code as one single layer of a 2D Abelian topological state. For simplicity of the discussion in this case, we will stick with the current description. One can check that with the particles of the form in Eq. (8) condensed, there is no deconfined particle in the 3D bulk when $p \equiv 1,2(\bmod 3)$. The absence of bulk deconfined particles can be directly verified by exhausting the possible forms of bulk particles and by noticing that all the particles that are not confined can be viewed as composite particles of the condensed ones. In Sec. III B, we will introduce a more general argument that leads to the same conclusion when applied to this case. When $p \equiv 1,2(\bmod 3)$, the only deconfined particles live on the open surface. The surface deconfined particles take the form

$\alpha_{1}(1,-1)^{T} \otimes e_{1}+\alpha_{2}\left[(1,0)^{T} \otimes e_{1}+(1,-1)^{T} \otimes e_{2}\right]$,

where $\alpha_{1,2}=1,2, \ldots, p$. Therefore, we can use a twocomponent integer vector $\alpha=\left(\alpha_{1}, \alpha_{2}\right)$. The two generators of these deconfined surface particles are shown in Fig. 2(b). The topological spin of the particle $\alpha$ can be calculated using the original $K$ matrix:

$$
\begin{aligned}
\theta_{\alpha} & =-\frac{2 \pi}{p}\left(\alpha_{1}^{2}+\alpha_{1} \alpha_{2}+\alpha_{2}^{2}\right) \\
& =\pi \alpha^{T} M \alpha
\end{aligned}
$$

where the second line is written in a matrix form with

$$
M=-\frac{1}{p}\left(\begin{array}{ll}
2 & 1 \\
1 & 2
\end{array}\right) .
$$

The braiding statistics of particles $\alpha$ and $\beta$ is given by

$$
\begin{aligned}
\theta_{\alpha \beta} & =-\frac{2 \pi}{p}\left(2 \alpha_{1} \beta_{1}+\alpha_{1} \beta_{2}+\alpha_{2} \beta_{1}+2 \alpha_{2} \beta_{2}\right) \\
& =2 \pi \alpha^{T} M \beta .
\end{aligned}
$$

Since all the surface particles are Abelian, their quantum dimensions are $d_{\alpha}=1$. There are $p^{2}$ deconfined particles on the surface. We expect the surface topological order to have total quantum dimension $\mathcal{D}=p$. For a $2 \mathrm{D}$ topological state, the chiral central charge $c$ is related to the quantum dimension and topological spins by the following formula [59]:

$$
\mathcal{D} e^{2 \pi i c / 8}=\sum_{\alpha} d_{\alpha} e^{i \theta_{\alpha}}
$$

Therefore, we can calculate the chiral central charge for these surface topological orders with $\mathcal{D}=p$ :

$$
\begin{aligned}
& c \equiv 0(\bmod 8), \quad \text { for } p \equiv 1(\bmod 3), \\
& c \equiv 4(\bmod 8), \quad \text { for } p \equiv 2(\bmod 3) .
\end{aligned}
$$

From this result, we see that, for $p \equiv 2(\bmod 3)$, the surface topological state has to have chiral topological order. This result is quite nontrivial because we start with layers of $Z_{p}$ toric code that are nonchiral. A similar situation can also be found using the Walker-Wang construction [32,33]. Also, for the special case with $p=2$, the resulting chiral surface topological state is the so-called $Z_{2}$ three-fermion state with chiral central charge $c=4$. When realized in $2 \mathrm{D}$, the corresponding $K$ matrix is the Cartan matrix of $S O(8)$ :

$$
K_{S O(8)}=\left(\begin{array}{cccc}
2 & -1 & -1 & -1 \\
-1 & 2 & 0 & 0 \\
-1 & 0 & 2 & 0 \\
-1 & 0 & 0 & 2
\end{array}\right) .
$$

This specific state is also a realization of the symmetrypreserving surface topological state of a time-reversalinvariant bosonic topological insulator [46]. To our knowledge, the chiral topological order for the cases with $p>2$ has not been well studied before.

The case with $p \equiv 0(\bmod 3)$ requires a more careful treatment. The set of quasiparticles that are deconfined on the surface is still given by Eq. (12), but the naive application of Eq. (13) will fail in this case. That is because other than the trivial particle $\alpha=(0,0)$, there are two other particles $(p / 3, p / 3)$ and $(2 p / 3,2 p / 3)$ that do not have any nontrivial braiding statistics with any other surface deconfined particles. Therefore, the surface theory is not modular if we include all $p^{2}$ particles labeled by $\left(\alpha_{1}, \alpha_{2}\right)$ with $\alpha_{1,2}=1, \ldots, p$. The simplest way to remedy this problem is to take the quotient of the $p^{2}$ particles by the three local particles $(0,0),(p / 3, p / 3)$, and $(2 p / 3,2 p / 3)$. Then, we end up with a theory of $p^{2} / 3$ particles, namely, the total quantum dimension $\mathcal{D}=p / \sqrt{3}$. Using the formula [Eq. (13)], we obtain that 


$$
c \equiv-2(\bmod 8), \quad \text { for } p \equiv 0(\bmod 3) .
$$

Again, we see that the surface topological order is chiral. For the simplest case with $p=3$, the surface topological order of the quotient theory is the time-reversal copy of the $U(1)_{3}$ Chern-Simons theory with the $K$ matrix given by

$$
K_{p=3}^{\text {surf }}=-\left(\begin{array}{ll}
2 & 1 \\
1 & 2
\end{array}\right) .
$$

In fact, rather than just a mathematical trick, the quotient does carry physical meanings. The particles that are mod out from the surface theory are a subgroup of particles generated by $(p / 3, p / 3)$. Written in the stacked-layertheory language, $(p / 3, p / 3)$ takes the form

$$
(2 p / 3,-p / 3)^{T} \otimes e_{1}+(p / 3,-p / 3)^{T} \otimes e_{2} .
$$

In fact, we notice that the particles $(2 p / 3,-p / 3)^{T} \otimes e_{n}+$ $(p / 3,-p / 3)^{T} \otimes e_{n+1}$ for $\forall n$ are deconfined. Therefore, we should identify them as one type of bulk deconfined point particle (at different positions in the $z$ direction). This deconfined bulk particle will become the one of the constituents of the 3D bulk topological order. Therefore, when we consider the surface topological order, this type of particle should not be included. In the next section, we will come back to this example to study its bulk topological order. To summarize this series of examples, we have obtained the surface topological order with

$\mathcal{D}=\frac{p}{\sqrt{3}} \quad$ and $\quad c \equiv-2(\bmod 8), \quad$ for $p \equiv 0(\bmod 3)$,

$\mathcal{D}=p \quad$ and $\quad c \equiv 0(\bmod 8), \quad$ for $p \equiv 1(\bmod 3)$,

$\mathcal{D}=p \quad$ and $\quad c \equiv 4(\bmod 8), \quad$ for $p \equiv 2(\bmod 3)$.

For $p \equiv 1,2(\bmod 3)$, the $3 \mathrm{D}$ bulk is a trivial $3 \mathrm{D}$ gapped state. For $p \equiv 0(\bmod 3)$, the bulk state has $3 \mathrm{D}$ topological order. It would be interesting to work out the effective $K$ matrix for the surface topological states for all $p$, especially the chiral ones, but finding this effective $K$ matrix is beyond the scope of this paper and will be left for future works.

\section{B. General criteria for 3D states with purely surface topological order}

In Sec. III A, we have given a series of examples [for $p \equiv 1,2(\bmod 3)]$ of $3 \mathrm{D}$ states with $2 \mathrm{D}$ surface topological order and trivial 3D bulk topological order using the layer construction. In this part of the discussion, we will provide general criteria for the construction of 3D states with purely surface topological order and trivial bulk topological order.

We denote the quasiparticle lattices generated by $\left\{p_{i}\right\}$, $\left\{q_{i}\right\}$ and $\left\{p_{i}\right\} \cup\left\{q_{i}\right\}$ as $\Gamma_{\left\{p_{i}\right\}}, \Gamma_{\left\{q_{i}\right\}}$ and $\Gamma_{\left\{p_{i}\right\} \cup\left\{q_{i}\right\}}$, and the local-particle lattice generated by the column vectors of the $K$ matrix $K$ as $\Gamma_{K}$. Notice that the lattice of all quasiparticles is given by the integer lattice $\mathbb{Z}^{r}$. Then, we have the following theorem.

Theorem 1.-If $\Gamma_{\left\{p_{i}\right\} \cup\left\{q_{i}\right\}}=\mathbb{Z}^{r}$ modulo $\Gamma_{K}$ and the overlap of $\Gamma_{\left\{p_{i}\right\}}$ and $\Gamma_{\left\{q_{i}\right\}}$ is trivial, i.e., $\Gamma_{\left\{p_{i}\right\}} \bigcap \Gamma_{\left\{q_{i}\right\}}=\varnothing$ modulo $\Gamma_{K}$, the resulting 3D states from the layer construction have only 2D surface topological order and trivial 3D topological order.

One can check that the examples discussed in the previous subsection satisfy the condition of this theorem when $p=1,2$ and, therefore, only admits purely surface topological order. The proof of this theorem is the following. Suppose we start with a stack of $L$ layers and turn on the coupling between the layers (assuming open boundary conditions in the $z$ direction) to form a 3D gapped state, as described in Sec. II. As is discussed in Sec. II, the 3D states from the layer construction always have a deconfined surface particle if we consider the open boundary condition. Therefore, we only need to prove that there is no nontrivial deconfined excitation in the bulk and thus no bulk topological order. If there is a deconfined particle living in the $m_{1}$ th layer and the $m_{2}$ th layer in the bulk, we can always denote it as $X_{m_{1}, m_{2}}=\sum_{m=m_{1}}^{m_{2}} x_{m} \otimes e_{m}$ with $1 \leq m_{1} \leq m_{2} \leq L$. Without the loss of generality, we can also assume that in this notation, $x_{m} \neq 0$. There are four possible situations that need to be discussed separately: (i) $1<m_{1} \leq m_{2}<L$, (ii) $1=m_{1} \leq m_{2}<L$, (iii) $1<m_{1} \leq$ $m_{2}=L$, and (iv) $\left(m_{1}, m_{2}\right)=(1, L)$. We are going to rule out the four possibilities one by one.

For situation (i), if there exists a deconfined particle $X_{m_{1}, m_{2}}$ with $1<m_{1}, m_{2}<L$ for the open boundary (in the $z$ direction) system, this particle will stay deconfined even when one introduces the the coupling between the top and bottom layers by the condensation of the composite particles $p_{i} \otimes e_{L}+q_{i} \otimes e_{1}, i=1, \ldots, r / 2$. However, since $\Gamma_{\left\{p_{i}\right\}} \cap \Gamma_{\left\{q_{i}\right\}}=\emptyset$ modulo $\Gamma_{K}$, all condensed null particles $\left\{n_{i}^{(m)}\right\}_{i=1, \ldots, r / 2}^{m=1, \ldots, L}$ for the periodic system are linearly independent. The number of these condensed null particles is equal to $\operatorname{dim}(\mathcal{K}) / 2$. Thus, from the results in Refs. [52-54], there should be no deconfined particle (other than the condensed ones) left in the condensate. Therefore, deconfined particles $X_{m_{1}, m_{2}}$ with $1<m_{1}, m_{2}<L$ should not exist.

For situation (ii), we consider deconfined particles of the form $X_{1, m 2}$ with $m_{2}<L$. The trivial statistics between $X_{1, m_{2}}$ and the condensed particles, especially $n_{i}^{\left(m_{2}\right)}$ (see Fig. 3), implies that $x_{m_{2}}$, as a single-layer particle, braids trivially with all the $p_{i}$ 's. Since $\Gamma_{\left\{p_{i}\right\} \cup\left\{q_{i}\right\}}=\mathbb{Z}^{r}$ modulo $\Gamma_{K}$, we can expand $x_{m_{2}}$ in the basis of $\left\{p_{i}\right\} \cup\left\{q_{i}\right\}$ and write $x_{m_{2}}=\sum_{i}\left(a_{i}^{\left(m_{2}\right)} p_{i}+b_{i}^{\left(m_{2}\right)} q_{i}\right)$.

We will show that the $\sum_{i} a_{i}^{\left(m_{2}\right)} p_{i}$ part of this expansion is 0 modulo local particles. Since $x_{m_{2}}$ is a single-layer particle that has trivial braiding statistics with all the $p_{i}$ 's and all the $q_{i}$ 's and also braids trivially with the $p_{i}$ particles in the 


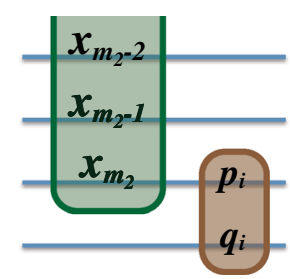

FIG. 3. In the discussion of situation (ii) (see the text), we consider the braiding between a deconfined particle $X_{1, m 2}$ (green region) with a condensed null particle $n_{i}^{\left(m_{2}\right)}$ (brown region).

single theory [see Eq. (4)], the single-layer particle $\sum_{i} a_{i}^{\left(m_{2}\right)} p_{i}$ should also braid trivially with all the particle $p_{i}$ 's. Therefore, from Eq. (4), we notice that the particles $\left(\sum_{i} a_{i}^{\left(m_{2}\right)} p_{i}\right) \otimes e_{m}$ with $\forall m$ are deconfined even for the system with periodic boundary conditions. As is explained in the previous situation, this deconfinement is impossible unless $\left(\sum_{i} a_{i}^{\left(m_{2}\right)} p_{i}\right) \otimes e_{m}$ 's are local particles.

Therefore, we can consider the fusion between the particle $X_{1, m_{2}}$ with the condensed particle $-\sum_{i} b_{i}^{\left(m_{2}\right)} n_{i}^{\left(m_{2}-1\right)}$, which does not change the nature of $X_{1, m_{2}}$ in the condensate. Now, notice that $X_{1, m_{2}}-$ $\sum_{i} b_{i}^{\left(m_{2}\right)} n_{i}^{\left(m_{2}-1\right)}$ is effectively a particle that lives in between the first and $\left(m_{2}-1\right)$ th layers and therefore can be identified as $\tilde{X}_{1, m_{2}-1}$. By iterating this argument, we can conclude that all deconfined quasiparticles for situation (ii) are equivalent to quasiparticles $X_{1,1}$ living at the top surface combined with a chain of condensed particles. Further, all the deconfined particles $X_{1,1}$ have to be decomposed into linear combinations of $q_{i}$ 's on the top surface. Using similar analysis, we can also show, for situation (iii), that the only possible nontrivial deconfined quasiparticles for situation (ii) are the $X_{L, L}$ 's and, therefore, are quasiparticles that live only on the bottom surface. Also, all the deconfined particles $X_{L, L}$ have to be decomposed into linear combinations of $p_{i}$ 's on the bottom surface.

For situation (iv), we focus on the deconfined particles of the form $X_{1, L}$. As is discussed above, we can decompose the $L$ th layer component $x_{L}$ of the $X_{1, L}$ into $x_{L}=\sum_{i}\left(a_{i}^{(L)} p_{i}+b_{i}^{(L)} q_{i}\right)$, so there exists a deconfined particle $X_{1, L-1}^{\prime}$ such that $X_{1, L}=X_{1, L-1}^{\prime}+\sum_{i} b_{i}^{(L)} n_{i}^{(L-1)}+$ $\sum_{i} a_{i}^{(L)} p_{i} \otimes e_{L}$. One can view this equation as the definition of $X_{1, L-1}^{\prime}$. The deconfinement of $X_{1, L-1}^{\prime}$ follows from the fact that $X_{1, L}$ and $\sum_{i} a_{i}^{(L)} p_{i} \otimes e_{L}$ deconfine in the open system, and $\sum_{i} b_{i}^{(L)} n_{i}^{(L-1)}$ is condensed. For the deconfined particle $X_{1, L-1}^{\prime}$, the discussion on situation (ii) already shows that, modulo the condensed particles, $X_{1, L-1}^{\prime}$ should be identified as $\tilde{X}_{1,1}^{\prime}$ and therefore as a deconfined particle on the top surface. Thus, the deconfined particle $X_{1, L}$ can always be identified as a trivial composition of a top- surface deconfined particle $\tilde{X}_{1,1}^{\prime}$ and a bottom-surface deconfined particle $\sum_{i} a_{i}^{(L)} p_{i} \otimes e_{L}$.

Combining the discussion from situations (i) to (iv), we conclude that when $\Gamma_{\left\{p_{i}\right\}} \bigcap \Gamma_{\left\{q_{i}\right\}}=\emptyset$ modulo $\Gamma_{K}$ and $\Gamma_{\left\{p_{i}\right\} \cup\left\{q_{i}\right\}}=\mathbb{Z}^{r}$ modulo $\Gamma_{K}$, the layer-constructed 3D state only admits nontrivial deconfined quasiparticles on its surface and therefore only admits surface topological order. The absence of a deconfined bulk quasiparticle indicates the trivialness of the topological properties in the 3D bulk. Also, the discussion of situations (ii) and (iii) further shows that, with the open boundary condition, the only possible excitation on the top (bottom) surface is given by the particle set $\Gamma_{\left\{q_{i}\right\}}\left(\Gamma_{\left\{p_{i}\right\}}\right)$. Therefore, the surface topological order on the top (bottom) surface is completely determined by the set $\Gamma_{\left\{q_{i}\right\}}\left(\Gamma_{\left\{p_{i}\right\}}\right)$ and the braiding statistics within it.

\section{Surface topological orders on different surfaces}

Having introduced the general criteria for 3D states with purely surface topological order from the layer construction, we will study the surface topological order on the top, bottom, and, especially, the side surfaces in greater detail in the following. As a method to build 3D gapped states, the layer construction is strongly anisotropic. This anisotropy will manifest itself when we consider a layer-constructed system with finite size in both the $z$ and $y$ directions. (Remember that the $x$ and $y$ directions are equivalent in our description.) The side surface appears to be significantly different from the top and bottom surfaces. We will show that, despite the superficial distinction among these surfaces, the surface topological orders on them are, in fact, equivalent. The implication of this result will be that the 3D states that result from the layer construction are topologically equivalent to an isotropic 3D gapped phase. Our discussion will still focus on the case with purely surface topological order.

We start by comparing the surface topological order on the top and bottom surfaces for the system with an open boundary along the $z$ direction. As is discussed in Sec. III B, quasiparticles of the surface topological order are given by $\Gamma_{\left\{q_{i}\right\}}$ for the top surface and by $\Gamma_{\left\{p_{i}\right\}}$ for the bottom surface. From the null condition $-p_{i}^{T} K^{-1} p_{j}=q_{i}^{T} K^{-1} q_{j}, \forall i, j$ in Eq. (4), we can establish a one-to-one correspondence between the sets $\Gamma_{\left\{q_{i}\right\}}$ and $\Gamma_{\left\{p_{i}\right\}}$, such that the braiding statistics in the set $\Gamma_{\left\{q_{i}\right\}}$ is exactly the conjugate of that in $\Gamma_{\left\{p_{i}\right\}}$. The braiding statistics discussed here is calculated with respect to a certain normal direction of the layers, which is the $+z$ direction in this case. The $+z$ direction is a natural choice of the normal direction of the top surface but is opposite to the normal direction of the bottom surface induced by the bulk. Consequently, taking the change of normal direction into account, we need to calculate the braiding statistics using the opposite $K$ matrix for the two surfaces. Therefore, the null condition 


$$
-p_{i}^{T} K^{-1} p_{j}=q_{i}^{T} K^{-1} q_{j}, \quad \forall i, j
$$

is exactly the condition for the equivalence between the topand bottom-surface topological orders.

Having established the equivalence of the top and bottom surfaces, we can move on to the discussion of the surface topological order on the side surface. First, let us briefly review the edge theory of a 2D Abelian topological state with a $K$ matrix $K$ and its behavior under quasiparticle condensation. The edge state of a 2D Abelian topological state with the $K$ matrix $K$ is described by the $1+1 \mathrm{D}$ chiral Luttinger-liquid theory [60]:

$$
\mathcal{L}_{\mathrm{CL}}=\frac{K_{I J}}{4 \pi} \partial_{x} \phi^{I} \partial_{t} \phi^{J}-V_{I J} \partial_{x} \phi^{I} \partial_{x} \phi^{J},
$$

where $V$ is the velocity matrix. Suppose the $2 \mathrm{D}$ bulk is in the condensed phase of a set of particles $\left\{n_{i}\right\}$ that satisfies $n_{i}^{T} K^{-1} n_{j}=0, \forall i, j$. The condensation is induced by turning on extra coupling terms in a chiral Luttinger liquid on the edge [52-54]:

$$
\delta \mathcal{L}_{\text {cond }}=\sum_{i}-g_{i} \cos \left(c_{i} n_{i}^{T} \phi\right),
$$

where $g_{i}$ 's are the coupling constants and $c_{i} \in \mathbb{Z}$ is the minimal integer, for each $i$, such that $e^{i c_{i} n_{i}^{T} \phi}$ only create or annihilate a local particle. Here, we have already organized the $\phi_{I}$ 's into a column vector $\phi$. Since $e^{i c_{i} n_{i}^{T} \phi}$ is a local operator, the $\delta \mathcal{L}_{\text {cond }}$ only involves local terms and, therefore, can be written as a local coupling using the microscopic degrees of freedom. Notice that the commutation relations between $n_{i}^{T} \phi$ 's (with different $i$ 's) are trivial in the chiral Luttinger-liquid theory:

$$
\left[n_{i}^{T} \phi, n_{j}^{T} \phi\right]=0, \quad \forall i, j .
$$

Therefore, in the deep condensation limit with $g_{i} \rightarrow \infty$, the coupling term $\delta \mathcal{L}_{\text {cond }}$ forces the fields $n_{i}^{T} \phi$ to develop nonzero vacuum expectation values:

$$
\left\langle n_{i}^{T} \phi\right\rangle \neq 0 .
$$

If the number of fields $n_{i}^{T} \phi$ equals $\operatorname{dim}(K) / 2$, then edge theory becomes fully gapped in the condensed phase.

Now, we will use this language to describe the topological order on the side surface of the layer-constructed 3D state with purely surface topological order. Before we introduce the condensation that couples the layers, the side surface of the stacked layer is described by the Lagrangian density:

$$
\begin{aligned}
\mathcal{L}_{\mathrm{CL}}^{\text {side }}= & \sum_{m} \frac{1}{4 \pi}\left(\partial_{x} \Phi^{(m)}\right)^{T} K\left(\partial_{t} \Phi^{(m)}\right) \\
& -\left(\partial_{x} \Phi^{(m)}\right)^{T} V\left(\partial_{x} \Phi^{(m)}\right),
\end{aligned}
$$

where $\Phi^{(m)}$ is the $r$-component [remember $r=\operatorname{dim}(K)$ ] chiral boson field on the edge of the $m$ th layer and $V$ is the velocity matrix for each layer. By quantizing this theory, we obtain the commutation relation of the boson fields:

$\left[\partial_{x} \Phi_{I}^{(m)}(x), \Phi_{J}^{(n)}(y)\right]=-i 2 \pi\left(K^{-1}\right)_{I J} \delta(x-y) \delta_{n, m}$.

The condensation of the particles $\left\{n_{i}^{(m)}\right\}$ that couple the layers also introduces the coupling in the chiral edge mode:

$$
\delta \mathcal{L}_{\text {cond }}=\sum_{i, m}-g_{m, i} \cos \left[l_{i}\left(p_{i}^{T} \Phi^{(m)}+q_{i}^{T} \Phi^{(m+1)}\right)\right],
$$

where $g_{m, i}$ 's are the coupling constants, and $l_{i} \in \mathbb{Z}$ is the minimal integer that ensures the locality of the operator $e^{i l_{i}\left(p_{i}^{T} \Phi^{(m)}+q_{i}^{T} \Phi^{(m+1)}\right)}$. That is to say, $e^{i l_{i}\left(p_{i}^{T} \Phi^{(m)}+q_{i}^{T} \Phi^{(m+1)}\right)}$ is the creation or annihilation operator of a local particle. It means that the layer construction can, in principle, be implemented by coupling the microscopic degrees of freedom without using nonlocal terms.

From the canonical quantization of the chiral Luttinger theory [Eq. (26)] and the null condition [Eq. (4)], we see that the fields in the cosine terms have trivial commutation relations:

$\left[\left(p_{i}^{T} \Phi^{(m)}+q_{i}^{T} \Phi^{(m+1)}\right),\left(p_{j}^{T} \Phi^{(n)}+q_{j}^{T} \Phi^{(n+1)}\right)\right]=0$,

$\forall i, j, m, n$.

Therefore, at the strong-coupling limit $g_{m, i} \rightarrow \infty$, the fields $\left(p_{i}^{T} \Phi^{(m)}+q_{i}^{T} \Phi^{(m+1)}\right)$ will develop a nontrivial vacuum expectation value, namely,

$$
\left\langle p_{i}^{T} \Phi^{(m)}+q_{i}^{T} \Phi^{(m+1)}\right\rangle \neq 0 .
$$

In the following, we will denote the field $p_{i}^{T} \Phi^{(m)}+$ $q_{i}^{T} \Phi^{(m+1)}$ as $\Lambda_{i}^{m+1 / 2}$.

Now, we can use this formalism to study the topological order on the side surface. From the Lagrangian density in Eqs. (26) and (28), we notice that we are, in fact, solving a problem of a coupled array of 1D wires. Similar coupledwire systems that exhibit 2D topological order were studied in Refs. [47-49]. The building blocks of these systems are $1 \mathrm{D}$ wires of normal Luttinger liquids. For our problem, we are considering the coupled arrays of fractionalized 1D wires that are the edge states of the 2D Abelian topological orders, as is shown in Fig. 4(a).

To identify the topological order on the side surface, we need to identify the nontrivial quasiparticles and their statistics. Deep in the condensed phase, the fields $\Lambda_{i}^{m+1 / 2}$ only fluctuate very weakly around its vacuum expectation values, which are pinned by the cosine terms in Eq. (28). In fact, the cosine terms create multiple degenerate minima for the fields $\Lambda_{i}^{m+1 / 2}$. The domain walls, or kinks, of $\left\langle\Lambda_{i}^{m+1 / 2}\right\rangle$ between different minima 
(a)

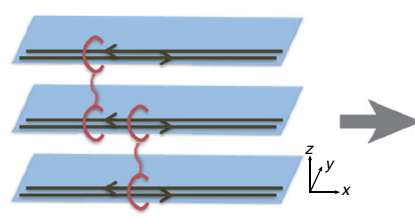

(b)

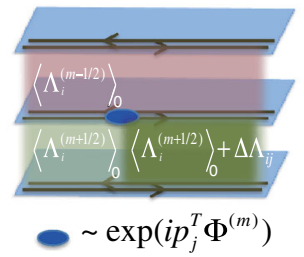

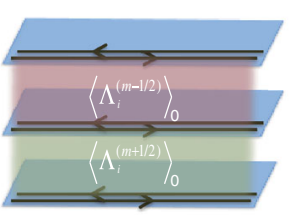

(c)

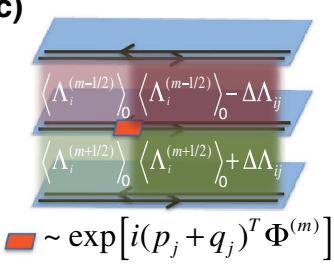

FIG. 4. (a) The condensation in the 3D bulk induces a coupling between the edge states of each layer. The side surface is effectively a coupled-wire system. In the strong-coupling limit, the vacuum expectation values $\left\langle\Lambda_{i}^{m+1 / 2}\right\rangle_{0}$ are pinned to the minima determined by the cosine terms in Eq. (28). The coloring on the right-hand side indicates nontrivial but uniform $\left\langle\Lambda_{i}^{m \pm 1 / 2}\right\rangle_{0}$ on the side surface. (b) The operator $e^{i p_{j}^{T} \Phi^{m}(x)}$ creates a collection of kinks (depicted as the change in color) in $\left\langle\Lambda_{i}^{m+1 / 2}(x)\right\rangle$, which will be identified as a topological quasiparticle $w_{j}$ on the $(m+1 / 2)$ th layer of the side surface. (c) The operator $\chi_{j}^{m}$ creates a kink-antikink pair that effectively tunnels the quasiparticle $w_{j}$ from the $(m-1 / 2)$ th layer to the $(m+1 / 2)$ th layer.

are topologically stable excitations and will be identified as topological quasiparticles on the side surface. We can start from the configuration with all $\left\langle\Lambda_{i}^{m+1 / 2}\right\rangle_{0}$ uniform. The operator $e^{i p_{j}^{T} \Phi^{(m)}(x)}$ creates a series of kinks in $\left\langle\Lambda_{i}^{m+1 / 2}\right\rangle, i=1, \ldots, r / 2$ [see Fig. 4(b)], such that the vacuum expectation values of $\left\langle\Lambda_{i}^{m+1 / 2}(x)\right\rangle$ on the right and left sides of $e^{i p_{j}^{T} \Phi^{(m)}(x)}$ differ by

$$
\Delta \Lambda_{i j}=2 \pi p_{i}^{T} K^{-1} p_{j}
$$

We will identify the $e^{i p_{j}^{T} \Phi^{(m)}(x)}$ as the creation operator of a quasiparticle, denoted by $w_{j}$, on the side surface. Since the kinks created by $e^{i p_{j}^{T} \Phi^{m}(x)}$ are the domain walls of $\left\langle\Lambda_{i}^{m+1 / 2}(x)\right\rangle$, we would like to think of this quasiparticle $w_{j}$ as residing in the $(m+1 / 2)$ th layer. The quasiparticle $w_{j}$ can tunnel between the $(m-1 / 2)$ th layer and the $(m+1 / 2)$ th layer, which is implemented by the tunneling operator

$$
\chi_{j}^{m}=e^{i\left(p_{j}^{T}+q_{j}^{T}\right) \Phi^{m}(x)} .
$$

The operator $\chi_{j}^{m}$ creates simultaneously a kink in $\left\langle\Lambda_{i}^{m+1 / 2}(x)\right\rangle$ and an antikink in $\left\langle\Lambda_{i}^{m-1 / 2}(x)\right\rangle$, as is shown in Fig. 4(c). It is straightforward to show that, in the strongcoupling limit,

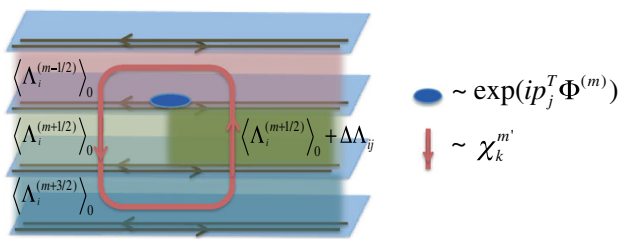

FIG. 5. This figure illustrates the braiding between two quasiparticles of the surface topological order on the side surface. The blue dot represents the quasiparticle $w_{j}$ created by the operator $e^{i p_{j}^{T} \Phi^{(m)}}$. The red line represents the trajectory of the second quasiparticle $w_{k}$ that the first one braids with. The operators that tunnel $w_{k}$ along the trajectory are explained in the main text. Especially, the vertical tunnel operator from the $m^{\prime}$ th layer to the $\left(m^{\prime}+1\right)$ th layer is given by $\chi_{k}^{m^{\prime}}$.

$$
\chi_{j}^{m+1} e^{i p_{j}^{T} \Phi^{(m)}(x)}=e^{i\left\langle\Lambda_{j}^{m+1 / 2}(x)\right\rangle} e^{i p_{j}^{T} \Phi^{(m+1)}(x)},
$$

which means that the operator $\chi_{j}^{m}$ tunnels the quasiparticle $w_{j}$ from the $(m-1 / 2)$ th layer to the $(m+1 / 2)$ th layer and, more importantly, with a phase factor $e^{i\left\langle\Lambda_{j}^{m+1 / 2}(x)\right\rangle}$. We will show that this phase factor will give rise to the braiding statistics of the quasiparticles on the side surface. We can consider the configuration with a quasiparticle $w_{j}$ on the $(m+1 / 2)$ th layer created by the operator $e^{i p_{j}^{T} \Phi^{(m)}(x)}$, as is shown in Fig. 5. A close path of the quasiparticle $w_{k}$ is depicted as the red line. The horizontal tunneling of the quasiparticle $w_{k}$ in the uniform backgrounds of $\left\langle\Lambda_{i}^{m-1 / 2}(x)\right\rangle_{0}$ and $\left\langle\Lambda_{i}^{m+3 / 2}(x)\right\rangle_{0}$ does not produce any nontrivial phase factor. The vertical tunneling between the layers through the operator $\chi_{k}^{m^{\prime}}$ 's, as is shown in Eq. (33), does carry nontrivial phase factors. Because of the existence of the kinks in $\left\langle\Lambda_{i}^{m+1 / 2}(x)\right\rangle$, the net phase, which is viewed as the braiding phase between the quasiparticles $w_{j}$ and $w_{k}$, is nonzero and is given by

$$
B_{j k}=e^{-i \Delta \Lambda_{j k}}=e^{-2 \pi i p_{j}^{T} K^{-1} p_{k}} .
$$

Therefore, by identifying the quasiparticle $w_{i}$ on the side surface with the deconfined particle $p_{i}$ on the bottom surface, we conclude that topological order on the side surface is the same as the one on the bottom surface. Furthermore, by noticing that the operators $e^{i p_{j}^{T} \Phi^{(m)}(x)}$ and $e^{-i q_{j}^{T} \Phi^{(m+1)}(x)}$ create exactly the same kink configuration on the side surface, we can also show the equivalence of the side surface and the top surface through the same analysis.

\section{3D TOPOLOGICALLY ORDERED STATES}

In this section, we will focus on the layer construction of 3D topologically ordered states. In Sec. III B, we have introduced the general criteria for the construction of a 3D gapped state with trivial bulk topological order. When this criterion is not satisfied, we will generically have bulk 
deconfined excitations, which will organize themselves to form 3D topological order. Unlike the topological order in $2 \mathrm{D}$, which is fully characterized by the modular tensorcategory theory, the general structure of 3D topological order is not yet known completely. The layer construction provides a method to construct 3D topological ordered gapped states that will shed light on the potential structure for a generic 3D topological order. In this section, we will consider three types of different examples of three-dimensional topological order. First, we will introduce the construction of conventional topological order with particle-string mutual statistics that resembles the $Z_{p}$ lattice gauge theory in three spatial dimensions. Second, we will construct an example with 3D topological order with coexisting surface topological order. Third, we will consider a more nontrivial example with braiding statistics not only between particles and strings but also between different types of strings.

\section{A. Conventional 3D topological order}

To construct a 3D gapped state with the conventional 3D topological order of $3 \mathrm{D} Z_{p}$ lattice gauge theory, we start with layers of $Z_{p}$ toric code with the $K$ matrix:

$$
K_{Z_{p}}=\left(\begin{array}{cc}
0 & p \\
p & 0
\end{array}\right) .
$$

We choose

$$
p_{1}=(1,0)^{T}, \quad q_{1}=-(1,0)^{T} .
$$

The condensed particles are illustrated in the Fig. 6(a). This condensation can be understood as an "exciton condensate," in which the electric particle in each layer, i.e., $(1,0) \otimes e_{m} \forall m$, is deconfined:

$$
\epsilon_{e}=(1,0)^{T} \otimes e_{m} .
$$

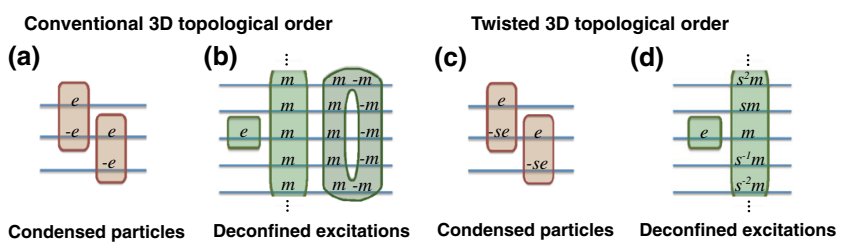

FIG. 6. (a) The condensation of $e$ and $-e$ composite in the two consecutive layers in the system of stacked layers of $Z_{p}$ toric codes. (b) The electric particle in each layer is a deconfined particle that should be identified as the electric particle in the 3D $Z_{p}$ lattice gauge theory. The string of $m$ particles in every layer is a deconfined string excitation that can be viewed as the flux string in the $Z_{p}$ gauge theory. The flux strings do not have to align strictly along the $z$ direction. A contractible flux string of finite size, as is shown in the figure, can be considered. (c) A different set of condensed particles in layers of $Z_{p}$ toric code, the condensation of which leads to the twisted lattice gauge theory (see the text). (d) The deconfined particles in the twisted theory, including an electric particle and the "twisted" flux string.
This electric particle $\epsilon_{e}$ can hop between the layers through the fusion with the condensed "excitons." Since spin of the electric particle $\epsilon_{e}$ is 0 , it is a boson in the 3D system. Besides the deconfined electric particle, the other type of deconfined excitation is the string excitation, which is composed of the magnetic particle $m$ in each layer, i.e.,

$$
\epsilon_{m \text { string }}=\sum_{m}(0,1)^{T} \otimes e_{m},
$$

as is shown in Fig. 6(b). So far, we have only considered the string excitations that orient along the $z$ direction. String excitations can, in fact, take, especially in the continuum limit, any orientations with their excitation energy proportional to their length. We can consider contractible strings of finite size as depicted in Fig. 6(b). From the $K$ matrix, it is easy to see that the braiding statistics between the electric particle and the magnetic string is

$$
\theta_{e, m \text { string }}=2 \pi / p \text {. }
$$

This statistical phase is independent from the location of the pointlike particle $\epsilon_{e}$. By comparing with the $3 \mathrm{D} Z_{p}$ lattice gauge theory, the deconfined electric particle can be identified as the electric particle in the $Z_{p}$ gauge theory, while the magnetic string can be identified as the magnetic flux string. These two types of deconfined excitation and their mutual statistics give rise to the topological order in 3D. In this example, the layer construction studies the anisotropic limit of the $Z_{p}$ gauge theory in which the $m$ string always orients itself along the $z$ direction. The string excitation can actually take arbitrary shape, and its energy is always linearly proportional to its length. The statistics between the point particles and string excitations should not depend on the shape of the string. Therefore, the layer construction faithfully captures the topological properties of 3D lattice gauge theory.

Now, we can consider a variation of this case with a topological twist in each layer. For the convenience of the discussion, we will assume that $p$ is a prime number such that $Z_{p}$ is a field, namely, $s^{-1} \in Z_{p}, \forall s \in Z_{p}$. In the following, we will use $s^{-1}$ to denote the integer whose product with $s$ is 1 modulo $p$. For a given $s \in Z_{p}$, we consider the condensate defined by

$$
p_{1}=(1,0)^{T}, \quad q_{1}=-(s, 0)^{T} .
$$

The condensed particles in this case are shown in Fig. 6(c). It is easy to see that the $e$ particle in each layer is still deconfined. It will be identified as a type of point particle in the 3D state. The hopping of $e$ particles between layers is still implemented through the fusion with the condensed particles. We see that in this hopping process, an $e$ particle of the $m$ th layer turns into an se particle of the $(m+1)$ th layer. We can understand this process as a hopping of the $e$ 
particle followed by a symmetric twist. To be more precise, for $Z_{p}$ toric code theory, the topological properties of the theory, namely, the braiding statistics and fusion rules, are invariant under the map generated by $e \rightarrow s e, m \rightarrow s^{-1} m$. This mapping is a symmetry of the $Z_{p}$ toric code as far as the topological properties are concerned. We will call this symmetry action the symmetric twist. The hopping process in this case is the hopping of the $e$ particle under one layer to the other followed by the symmetric twist $e \rightarrow s e, m \rightarrow s^{-1} m$.

Based on this understanding, one should expect that the topological order obtained in this case is the same as the untwisted case. Indeed, in this twisted case, there is a twisted version of the $m$ string [see Fig. 6(d)] that is given by

$$
\epsilon_{m \text { string }}^{\prime}=\sum_{m}\left(0, s^{-m}\right) \otimes e_{m}
$$

We pick the units of pointlike excitation and stringlike excitation to be the ones shown in Fig. 6(d). Their mutual braiding statistics is given by

$$
\theta_{e, m \text { string }}^{\prime}=2 \pi / p \text {. }
$$

It is the same as the untwisted case. Also, we see that the hopping of the $e$ particle between the layers is compatible with its braiding with the twisted $m$ string. So far, the analysis indicates that the local properties of the twisted case are the same as those of the untwisted one. However, this analysis is done for an infinite system or a system with open boundaries. When we consider periodic boundary conditions in the $z$ direction, the twisted case automatically generates a monodromy when we consider the hopping of the electric particle along the nontrivial cycle along the $z$ direction. To be more precise, an electric particle $e$ becomes the particle $s^{L} e$ after traversing along the $z$-direction cycle, where $L$ is the number of layers in the system. The twist generated by the condensed particle can be undone locally by considering the symmetric twist generated by $e \rightarrow s^{-l} e$ and $m \rightarrow s^{l} m$ for the $l$ th layer. Then, the system with a periodic boundary condition is equivalently described by the stacked layers of $Z_{p}$ toric code with the following condensed particles: $(1,0)^{T} \otimes e_{m}+(1,0)^{T} \otimes e_{m}+1$ with $m=1, \ldots, L-1$ and $\left(s^{-L}, 0\right)^{T} \otimes e_{L}+(1,0)^{T} \otimes e_{1}$. The system is locally equivalently to the untwisted case, and the twist along the $z$-direction cycle is generated by the coupling between the first and the $L$ th layers induced by the condensation of the particle $\left(s^{-L}, 0\right)^{T} \otimes e_{L}+$ $(-1,0)^{T} \otimes e_{1}$. Therefore, we can think of the twisted model as the conventional $3 \mathrm{D} Z_{p}$ gauge theory with a $2 \mathrm{D}$ membrane twist defect between the first and the $L$ th layers. (The notation of twist defects in 2D and their properties are first studied in Refs. [61,62]. Its generalization in 3D is first studied in Ref. [63].) This membrane defect implements the symmetry twist $e \rightarrow s^{L} e$ in the $3 \mathrm{D} Z_{p}$ gauge theory. Let us look at the ground-state degeneracy (GSD) of this twisted model on $T^{3}$. Assume that we also have periodic boundary conditions along the $x$ and $y$ directions. For the untwisted case, we can study the Wilson-loop operators $W_{x}, W_{y}$, and $W_{z}$ that tunnel the $e$ particle around the nontrivial cycles along the $x, y$, and $z$ directions. The Wilson-loop operators measure the magnetic flux in the nontrivial cycles and can take $p$ different values $e^{i 2 \pi n / p}, n=1, \ldots, p$ for the $Z_{p}$ gauge field. Therefore, the untwisted system has $p^{3}$ degenerate ground states on $T^{3}$. For the twisted case, $W_{z}$ is not well defined because the twist membrane renders the tunneling one cycle around the $z$ direction unclosed unless $s^{L} \equiv 1(\bmod p) . W_{x}$ and $W_{y}$ are still well defined. However, if we adiabatically move the tunneling path of $W_{x(y)}$ all together one cycle along the $z$ direction, the twist membrane generates a map: $W_{x(y)} \rightarrow W_{x(y)}^{s^{L}}$. It implies that the flux along the $x(y)$ direction measured by $W_{x(y)}$ should be invariant under the map and therefore can only take the flux value 1 unless $s^{L} \equiv 1(\bmod p)$. Therefore, when $s^{L} \not \equiv 1(\bmod p)$, the GSD on $T^{3}$ is 1 . For $s^{L} \equiv 1(\bmod p)$, the twist membrane only performs a trivial twist, which means the GSD on $T^{3}$ is the same as the untwisted case. These results are summarized as follows:

$$
\begin{aligned}
& \text { the GSD on } T^{3}=1, \quad \text { for } s^{L} \not \equiv 1(\bmod p), \\
& \text { the GSD on } T^{3}=p^{3}, \quad \text { for } s^{L} \equiv 1(\bmod p) .
\end{aligned}
$$

Therefore, we see that the twisted model has a different behavior of the ground-state degeneracy on $T^{3}$ from the untwisted case.

\section{B. Coexisting bulk and surface topological order}

In Sec. III A, we discussed a construction using layers of $Z_{p}$ toric codes with the condensed particles given by Eq. (8) [see Fig. 2(a)]. As is explained there, for $p$ dividable by 3 , there are two particles that have trivial statistics (except the vacuum particle), with all the rest of the surface deconfined particles. One of them can be written as

$$
(p / 3, p / 3)^{T} \otimes e_{1}+(-p / 3, p / 3)^{T} \otimes e_{2} .
$$

The other particle is a two-particle bound state of this one. [This form is essentially equivalent to the expression given in Eq. (19).] After we find the quotient of all the surface deconfined particles by these two particles, we can consistently calculate the central charge of the surface topological order. It implies that these two trivial particles should not be considered as part of the surface topological order. In fact, one can check that particles of the form

$\epsilon_{\mathrm{pt}}=(p / 3, p / 3)^{T} \otimes e_{m}+(-p / 3, p / 3)^{T} \otimes e_{m+1}$ 
are all deconfined. Therefore, this particle should be viewed as a bulk deconfined point particle, which is a generalization of the bulk deconfined electric particle in the previous example. Again, the spin of the particle $\epsilon_{\mathrm{pt}}$ is 0 , so it is a boson in the 3D system, which is consistent with the fact that any point particle in 3D should have either bosonic or fermionic statistics. Similarly to the previous example, there is also a type of deconfined string excitation that takes the simple form of

$$
\epsilon_{\mathrm{str}}=\sum_{m}(1,0)^{T} \otimes e_{2 m},
$$

which is shown in Fig. 7(b). Naively, there is another type of stringlike deconfined excitation that takes the form of $\sum_{m}(1,0)^{T} \otimes e_{2 m+1}$. However, it can be identified as $\epsilon_{\text {str }}$ fused with a string of condensed particles and, therefore, is considered as topologically equivalent to $\epsilon_{\mathrm{str}}$. The braiding statistical angle between the deconfined point particle and the string excitation is

$$
\theta_{\mathrm{pt}, \mathrm{str}}=2 \pi / 3 \text {. }
$$

Therefore, the bulk topological order of this case is equivalent to that of a $3 \mathrm{D} Z_{3}$ lattice gauge theory. However, as is discussed in Sec. III, this system also hosts a surface topological order with chiral central charge $c \equiv 2(\bmod 3)$ on its open boundary. Therefore, it is a layer-constructed system that has coexisting bulk and surface topological orders.

This situation can also be compared with the WalkerWang model [32]. The input data of the Walker-Wang model are a braided tensor category. If the braided tensor category (which describes a 2D topological order) is modular, namely, only the vacuum particle in this tensor category braids trivially with all the rest of the quasiparticles, the Walker-Wang model provides a construction of a 3D gapped state with trivial bulk topological order but nontrivial surface topological order characterized by the input modular tensor category. If the input data are only premodular, there will be, by definition, transparent (a)

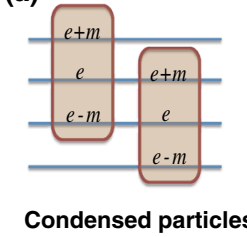

(b)

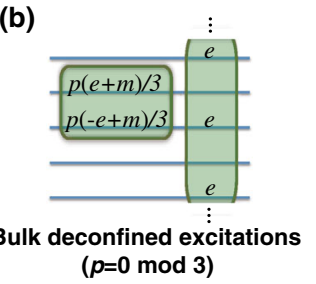

FIG. 7. (a) The condensed particle in the coupled $Z_{p}$ toric code system. This case is the same as the case studied in Sec. III A, but here, we focus on the cases with $p \equiv 0(\bmod 3)$. (b) The bulk deconfined excitations in this system. The one on the left is a point particle, while the one on the right is a string excitation. They have nontrivial mutual statistics. particles (other than the vacuum particle) that braid trivially with all other particles. In the Walker-Wang model associated with a premodular tensor category, the transparent particles can propagate in the bulk and become the quasiparticles of the 3D topologically ordered states. This scenario seems similar to the case of our layerconstructed model. For this model, with $p \equiv 1,2(\bmod 3)$, the surface topological order studied in Sec. III is modular and the 3D bulk has trivial topological order. For $p \equiv 0(\bmod 3)$, the deconfined particles on the surface only form a premodular tensor category. If we quotient out the surface particles that braid trivially with others, we get a consistent surface topological order. Those particles indeed penetrate in the bulk and form 3D topological order. Because of the similarity between these two situations, one might want to conjecture that the lattice model realizations of the layer construction in these cases can be formulated using the Walker-Wang model.

In the recently proposed general theory of topological order dimension [64], it is claimed that a certain type of anomaly for gapped states in $n$ space-time dimensions is classified by the topological order with gapped boundaries in $n+1$ space-time dimensions. This type of anomaly may be different from what we usually understand, since it is associated with gapped anomalous theories. Using this example of the layer-constructed system discussed above, we can understand this statement from Ref. [64]. The layerconstructed systems always have gapped surfaces. If there exist transparent particles, as is defined above, on the gapped surface, these transparent particles can tunnel into the bulk and become the constituents of the 3D bulk topological order. In this case, according to Ref. [64], the surface theory should be anomalous. In the example discussed above, this anomaly can be understood from various perspectives. First, if we look at all the particles that are deconfined on the surface (without distinguishing whether or not a particle is transparent), a naive calculation of the chiral central charge of the surface theory using Eq. (13) will show that the chiral central charge is a complex number that is anomalous for a consistent unitary modular tensor category. It is only after we take the quotient of the set of deconfined particles by the transparent ones that we obtain a consistent chiral central charge, as is shown in Eq. (17). Therefore, we notice that the transparent particles that should be identified as parts of the bulk 3D topological order are the source of the surface anomaly. The second perspective is briefly mentioned already in the discussion around Eq. (17). If we include all the particles and calculate the modular $S$ matrix of the surface theory, we will notice that the $S$ matrix is not unitary and thus make the surface theory not modular. As is expected, we can restore the modularity by the taking the quotient by the transparent particles. In all, we see in this example that when there exist transparent particles on the surface, or equivalently, when the bulk is topologically ordered, the the set of all surface 
deconfined particles forms an anomalous theory. In contrast, when the bulk is not topologically ordered [for $p \not \equiv 0(\bmod 3)]$, the surface theory is free of this anomaly. This anomaly we observed in this example is consistent with the claim in Ref. [64] that the anomaly of a gapped theory is associated with the topological order in one higher dimension.

\section{More generic 3D topological states with string braiding statistics}

Now, we will consider a more generic situation. The starting point is still stacked layers of $Z_{p}$ toric code. Here, we will first assume that $p$ is a multiple of 4 . For the convenience of later discussions, we view two layers of $Z_{p}$ toric codes as one single layer described by the $K$ matrix:

$$
K_{0}=\left(\begin{array}{llll}
0 & p & 0 & 0 \\
p & 0 & 0 & 0 \\
0 & 0 & 0 & p \\
0 & 0 & p & 0
\end{array}\right) .
$$

For later convenience, we will denote the four generators of all quasiparticles $(1,0,0,0)^{T},(0,1,0,0)^{T},(0,0,1,0)^{T}$, and $(0,0,0,1)^{T}$ as $c_{1}, f_{1}, c_{2}$, and $f_{2}$. The condensed particles (see Fig. 8) are given by the data:

$$
\begin{aligned}
& p_{1}=(1,0,2,0)^{T}, \quad q_{1}=(-1,0,-2,0) ; \\
& p_{2}=(1,2,0,-1)^{T}, \quad q_{2}=(1,-2,0,1) .
\end{aligned}
$$

In this condensate, there are two types of deconfined point particles

$$
\begin{aligned}
\epsilon_{c \mathrm{pt}} & =(1,0,2,0)^{T} \otimes e_{m}, \\
\epsilon_{f \mathrm{pt}} & =(0,0,0, p / 2)^{T} \otimes e_{m}, \quad \forall m,
\end{aligned}
$$

as is shown in Fig. 8(b). These point particles can hop between the layers by fusion with condensed particles.

(a)

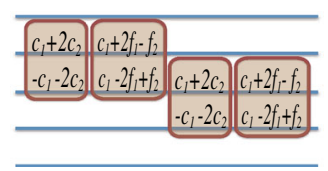

Condensed particles

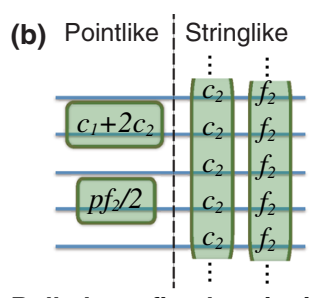

Bulk deconfined excitations
FIG. 8. (a) The condensed particles for a more generic layerconstructed 3D state. Each layer contains two identical copies of $Z_{p}$ toric code, with $p$ a multiple of $4 . c_{1,2}$ and $f_{1,2}$ are the charge and flux particles in the two $Z_{p}$ toric code systems, respectively. (b) The set of deconfined excitations, including two types of point particles and two types of string excitations with nontrivial mutual braiding.
Their mutual statistics is trivial. There are also two different types of string excitations [Fig. 8(b)]:

$$
\begin{aligned}
& \epsilon_{c \text { str }}=\sum_{m}(0,0,1,0)^{T} \otimes e_{m}, \\
& \epsilon_{f \text { str }}=\sum_{m}(0,0,0,1)^{T} \otimes e_{m} .
\end{aligned}
$$

The braiding statistics between the point particles and string excitations are given by

$$
\theta_{I, J}=2 \pi\left(\begin{array}{cc}
2 / p & 0 \\
0 & 1 / 2
\end{array}\right)
$$

where $I=1,2$ stand for point particles $\epsilon_{c \mathrm{pt}}$ and $\epsilon_{f \mathrm{pt}}$, respectively, while $J=1,2$ stand for string excitations $\epsilon_{f \text { str }}$ and $\epsilon_{c \text { str }}$. More interestingly, from the expressions of $\epsilon_{c \text { str }}$ and $\epsilon_{f \text { str }}$, it seems that these string excitations can also have nontrivial mutual braiding statistics. Indeed, if the system is periodic along the $z$ direction, the two types of strings can wrap around the $z$ direction and their mutual braiding phase is given by $\omega_{c \mathrm{str}, f \mathrm{str}}=2 \pi L / p$, where $L$ is the number of layers in the system. This result can simply be obtained by looking the system from the $z$ direction and viewing it as a quasi-2D system. From this point of view, the two string excitations are simply point particles in the 2D sense. Their braiding is also understood as the braiding of particles in 2D. This analysis only applies to vertical strings that wrap around the $z$-direction cycle. We need to inspect further the string braiding statistics for strings with finite size.

The strings with finite size can be constructed in the same way as we did in Sec. IV A. Figure 9(a) illustrates the construction of contractible strings of finite size for the example system. Each plane represents a layer in the layer construction. The particle contents of the string excitations in each layer are shown. For example, the blue string in Fig. 9(a) represents the finite-size version of the deconfined excitation $\epsilon_{f \text { str }}$, which is given by a line with $c_{2}$ in each of the five layers together with another line of $-c_{2}$ in each of the five layers. In general, an oriented string is defined as a chain of particles by the following rule. When the string crosses a plane and the direction of the string is parallel (antiparallel) to the normal direction of the plane (which is the $\hat{z}$ direction in our example), a $c_{2}\left(-c_{2}\right)$ particle is assigned to the crossing point. The same definition applies to the other type of string, except for a substitution of $c_{2}$ with $f_{2}$. In the following, we will refer to the string consisting of $c_{2}$ particles as the $c$ string and the other type as the $f$ string. It is straightforward to see that these strings are deformable. The deformation of string along the $x y$ plane is simply the change of position of $c_{2}$ 's or $f_{2}$ 's within the layers. The deformation along the $z$ direction is achieved by pair creation or pair annihilation of $c_{2}$ 's or $f_{2}$ 's. For example, if we fuse the $c_{2}$ and $-c_{2}$ of the $f$ string 
(a)

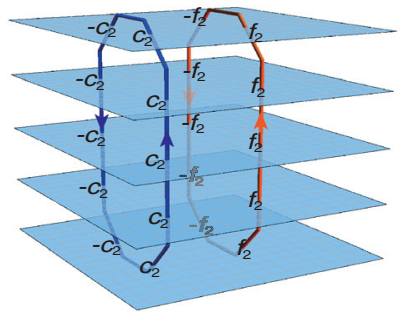

(b1)

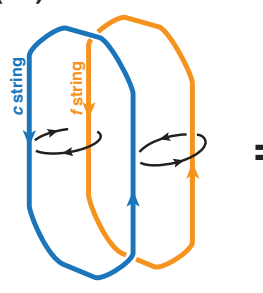

(b2)

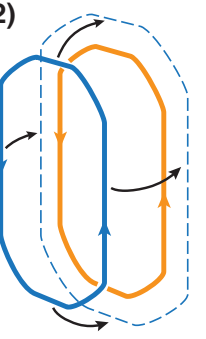

(b3)

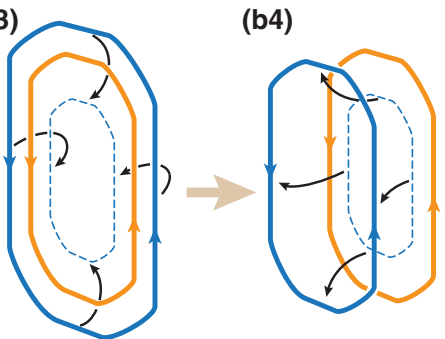

FIG. 9. (a) The definition of a $c$ string (blue line) and an $f$ string (orange line). Each plane represents a layer of the 2D topological state. The convention on the orientation of the string is explained in the main text. (b1) The braiding process of the two strings. (b2)-(b4) The braiding process shown in decomposed steps.

in the bottom layer in Fig. 9(a), the string effectively shrinks to a smaller size.

The braiding between two types of strings is defined to be the process shown in Figs. 9(b1)-9(b4). Figure 9(b1) is the overall action to the $c$ string (blue line) to complete the braiding with the $f$ string (orange line). For the sake of clarity, we have shown the decomposed steps in this action in Figs. 9(b2)-9(b4). First, we expand the $c$ string and move it past the $f$ string such that the $f$ string effectively passes through the "inside" of the $c$ string [Fig. 9(b2)]. Then, we shrink the $c$ string and move it back through the inside of the $f$ string [Fig. 9(b3)]. Once the $c$ string passes through the $f$ string, we expand the $c$ string back to its original size and move it back to the original position [Fig. 9(b4)]. In fact, it is straightforward to show that this type of braiding is always trivial for a contractible $c$ string and $f$ string. One can verify it by considering the total phase that results from the braiding of the particles $c_{2}$ 's and $f_{2}$ 's in each layer during the braiding of strings. The braiding phase between all the $c_{2}$ 's with all the $f_{2}$ 's is exactly canceled by that between all the $-c_{2}$ 's and all the $-f_{2}$ 's due to the opposite directions of the two braids. This trivial braiding statistics of two contractible strings is consistent with the observation of Ref. [41] that the braiding between two contractible strings must be trivial.

In order to obtain some nontrivial string braiding statistics, we need to consider a system with an edge dislocation and the $c$ string and $f$ string that are linked with the edge dislocation [see Fig. 10(a)]. In the following, we will refer to the edge dislocation with a unit Burgers vector as the $d$ string. The orientation of the $d$ string is determined through the right-hand rule by the normal direction of the surface that ends at the $d$ string. Now, we can study the braiding process between the $c$ string and the $f$ string, both of which are linked to a $d$ string. Figure 10(b1) shows the overall action to the $c$ string (blue line) in the braiding process at the presence of the $d$ string. Figures 10(b2)-10(b4) show the decomposed steps for the braid. (Similar threestring braiding processes in $Z_{N}^{k}$ or $Z_{N_{1}} \times Z_{N_{2}} \times Z_{N_{3}} \times Z_{N_{4}}$ gauge theory are considered in Refs. [41-43].) In this process, the string braiding statistics, which is given by the total braiding phase between the quasiparticles that form these strings, is

$$
\omega_{c, f}^{d}=\theta_{c_{2}, f_{2}}=2 \pi / p
$$

where $\theta_{c_{2}, f_{2}}$ is the braid statistics of the $c_{2}$ and $f_{2}$ particles in a single layer of the 2D topological state defined by the $K$ matrix $K_{0}$. More generally, if we consider a composite string of $n_{c} c$ strings and that of $n_{f} f$ strings that are both linked with an edge dislocation with a Burgers vector of length $b$, the string braiding statistics is given by

$$
b n_{c} n_{f} \omega_{c, f}^{d}=2 \pi n_{c} n_{f} b / p .
$$

From Fig. 10(a), it is easy to see that the net contribution to this phase $\omega_{c, f}^{d}$ is essentially given by the braiding between the $c_{2}$ particle and the $f_{2}$ particle in the defected layer. Compare to the braiding statistics between point particles and string excitations in Eq. (52); when $p$ is a multiple of 4, (a)

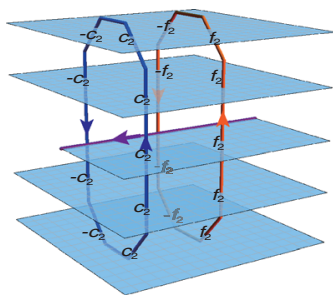

(b1)

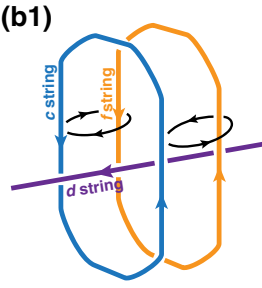

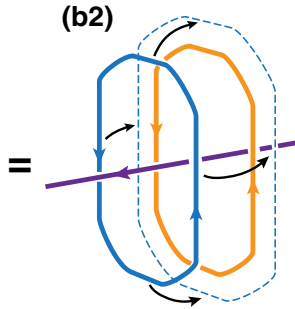

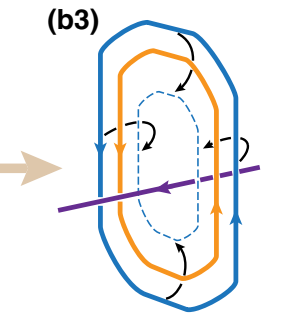

(b4)

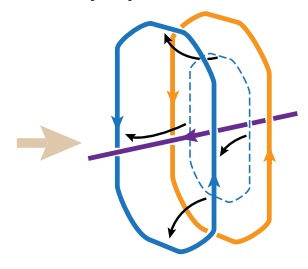

FIG. 10. (a) The configuration of the $c$ string and the $f$ string that link with an edge dislocation (or a $d$ string) which is indicated by the purple line. (b1) The braiding process of the $c$ string and the $f$ string that are both linked with the $d$ string. (b2)-(b4) The decomposed steps in this braiding process. 
the string braiding statistics $\omega_{c, f}^{d}$ is more fractionalized. Thus, it cannot be removed by attaching point particles to the strings. Also, the fact that the $d$ string is an extrinsic defect rather than a dynamical one does not undermine the topological protection of the string braiding statistics $\omega_{c, f}^{d}$ either. That is because throughout the braiding process, the $d$ string does not have to move, which means that no additional dynamical phase because of the extrinsic nature of the $d$ string should come into play. Furthermore, the three-string braiding phase $\omega_{c, f}^{d}$ can be related to the braiding phase $\omega_{c \text { str, } f \text { str }}=2 \pi L / p$ between two vertical strings winding around the torus, which we discussed at the beginning of this subsection. An $L$-layer system can be formally considered as a zero-layer system (vacuum) with an edge dislocation in the $x y$ plane at infinity with a Burgers vector $L \hat{z}$, i.e., $L d$ strings at infinity. With the periodic boundary condition, the $c$ strings and the $f$ string that wrap around the nontrivial cycle in the $z$ direction, in fact, have a nontrivial linking number with all of the $L d$ strings and therefore have nontrivial string

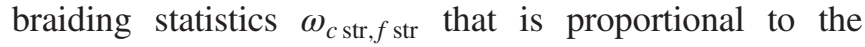
number of layers $L$.

There is an alternative approach to compute the stringstring braiding statistics, which will be helpful for our later discussion. To explain this approach, we consider the string fusion and splitting process in a similar way as in Ref. [41]. When we consider our system as a multilayer 2D system, the finite-size $c$ string and $f$ string are special configurations of quasiparticles that live in some number of layers. Therefore, their deformation and fusion can be understood in terms of the fusion of quasiparticles in the 2D theory. The specific type of deformation that we are interested in is the deformation that changes the number of strings. Here, we will show that the string excitations in the layer-constructed system can also deform, and especially split and fuse, without accumulating the nontrivial Berry's phase. To demonstrate the deformation of the strings, we can consider the example shown in Fig. 11. One $c$ string can be split into two independent $c$ strings by bringing two different points on the string very close to each other and pinching this point off. In this process, we use nothing more than the fusion rules of quasiparticles in each layer. In the current setting, $c_{2}$ in each layer is an Abelian particle with no spin.
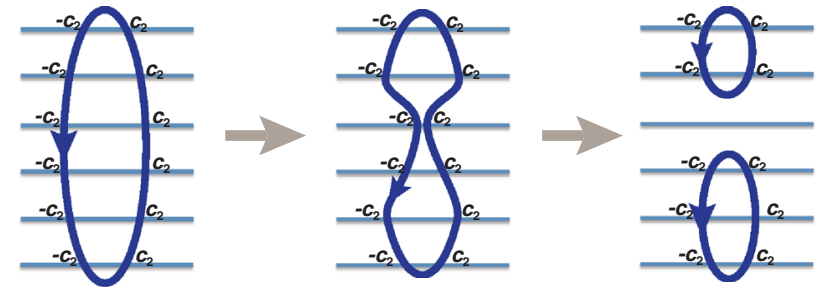

FIG. 11. One $c$ string can be deformed and split into two $c$ strings through the fusion of $c_{2}$ 's within the layers. The reverse process can be viewed as the fusion between two $c$ strings.
Therefore, the splitting process does not have any nontrivial Berry's phase associated with it. One can also consider the reverse process of splitting, which is the fusion of two $c$ strings. The same analysis is also applicable to the $f$ strings.

Since for each layer of the 2D topological state the fusion and braiding of quasiparticles are operations that commute with each other, we argue that the string braiding also commutes with string deformation, including the splitting and fusion of strings. To be more precise, we can deform, split, and fuse strings without changing the total Berry's phase in the braiding of string as long as the overall process remains topologically equivalent. Using this argument, we can obtain a new view to the braiding process [Fig. 10(b1)] that we discussed above. The braiding process is equivalent to the process shown in Figs. 12(a)-12(d). First, we pick an infinitesimal segment [the red-shaded area in Fig. 12(a)] of the $c$ string and braid it around the $f$ string without moving the rest of the $c$ string. This action creates locally a link (yellow region) and an antilink (gray region) [see Fig. 12(b)]. The link and antilink can annihilate each other if we bring them close together. Now, instead of annihilating them together directly, we bring the local link in the yellow region around the $d$ string [Fig. 12(b)]. We then annihilate the yellow and the gray regions by bringing them together afterward [Fig. 12(c)]. The whole process shown in Figs. 12(a)-12(d) is topologically equivalent to the braiding of strings defined in Fig. 10(b1). We argue that in the combined processes of Figs. 12(a)-12(d), only the process in Fig. 12(b) contributes to the nontrivial Berry's phase. It is because (1) the process shown in Fig. 12(a) only moves an arbitrarily small segment and should not accumulate any nontrivial Berry's phase and (2) the annihilation between the yellow and gray regions also does not contribute to the Berry's phase since it can also be thought of as bringing an infinitesimal segment of the $c$ string around the $f$ string as we did in Fig. 12(a). Given that all of the nontrivial Berry's phase comes from the process in Fig. 12(b), we will reinterpret it using the fusion and splitting of strings. As is shown in Fig. 12(i), the strings in the yellow region can be deformed, upon splitting and fusion, to unlinked $c$ strings and $f$ strings accompanied by a link $L_{c, f}$ of linking number 1 between the $c$ string and the $f$ string. Therefore, the process shown in Fig. 12(b) is equivalent to the combined processes shown in Figs. 12(e)-12(h). Since the fusion and splitting do not produce a nontrivial Berry's phase in this process, the total Berry's phase is equal to the Berry's phase $\Omega_{L_{c, f}}^{d}$ of braiding the link $L_{c, f}$ around the the $d$ string. The analysis above can be summarized by the equation

$$
\omega_{c, f}^{d}=\Omega_{L_{c, f}}^{d}
$$

In the analysis above, for the sake of generality, we have implicitly assumed that the $c$ strings and $f$ strings can be considered as continuous objects in the space. We expect 
(a)

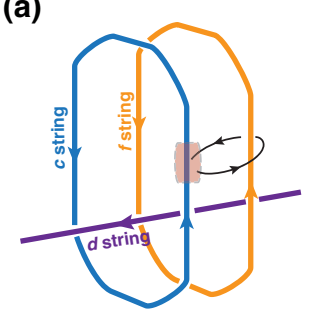

(b)

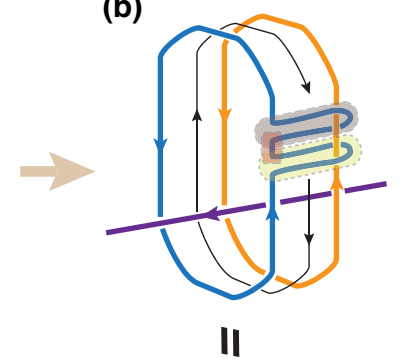

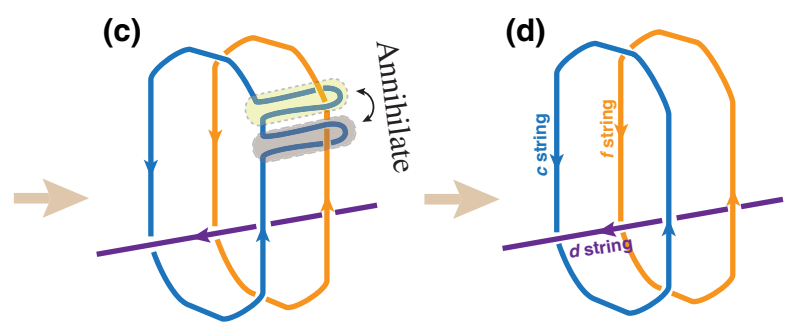

Equivalent process to (b)

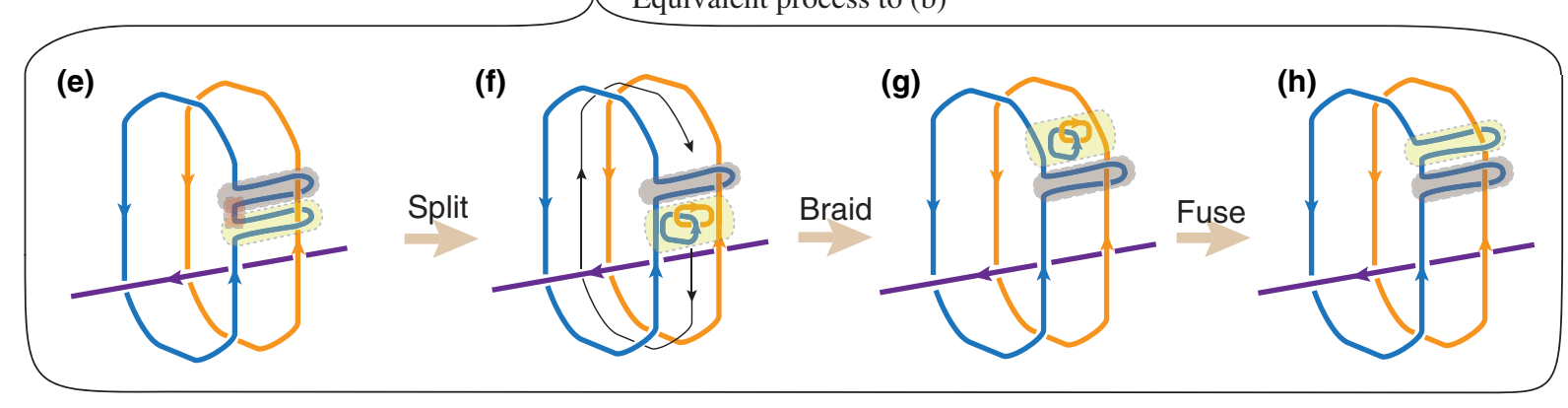

(i)

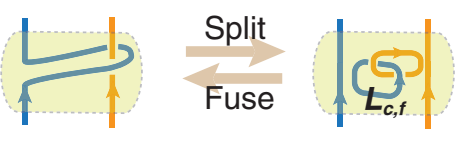

(j)

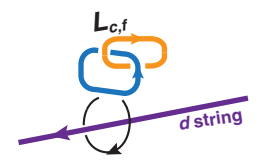

FIG. 12. (a)-(d) An alternative string braiding process that is topologically equivalent to that shown in Fig. 10. Upon fusion and splitting of the strings, the process in (b) can be deformed to the combined process shown in (e)-(h). (i) The local identification of string configuration under splitting and fusion, which plays a central role in the reinterpretation of the process shown in (b). The total Berry's phase in the string braiding process equals the Berry's phase in the process induced by the braiding of the link $L_{c, f}$ around the $d$ string, as is shown in $(\mathrm{j})$.

that its validity still holds even when the space is discrete. In fact, we can verify Eq. (55) by explicitly calculating $\Omega_{L_{c, f}}^{d}$ in our layer-constructed system. We start by studying the Berry's phase induced by the local motion of the link $L_{c, f}$. If we hold the relative positions of the $c$ string and the $f$ string in the link $L_{c, f}$ fixed, it is obvious that moving $L_{c, f}$ along the $x$ or the $y$ direction does not produce any nontrivial phase. In contrast, moving the $L_{c, f}$ in the $z$ direction while keeping the relative positions of the $c$ string and the $f$ string fixed will induce a nontrivial Berry's phase. The link configuration of $L_{c, f}$ is drawn in Fig. 13(a). The orientations of the strings are indicated by the arrows of the corresponding colored circles (blue and orange). The layers that are supposed to be represented by horizontal lines are omitted in this figure, and the $z$ direction is indicated by the black arrow. Let us consider moving this configuration by one layer down toward the $-z$ direction. For the parts outside the gray-shaded region in Fig. 13(a), since there is no nontrivial linking between these segments of the $c$ string and the $f$ string, they can be deformed into the configuration that is one layer below their original position without inducing any Berry's phase. In the gray-shaded region, the linked parts of the $c$ string and the $f$ string need to be treated more carefully. To move the linked part one layer toward the $-z$ direction, we essentially have to rotate the part of the link enclosed by the dashed gray line in Fig. 13(b) by $\pi$ about the $z$ axis. The net Berry's phase associated with this rotation is equivalent to a full braid between $c_{2}$ and $f_{2}$ in a single layer and is, therefore,

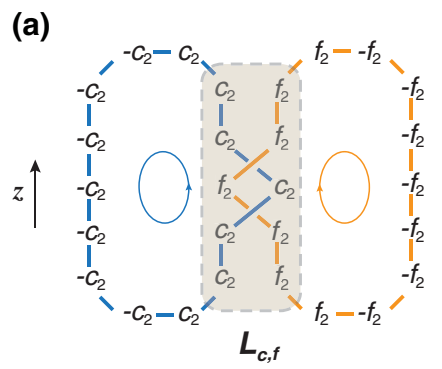

(b)

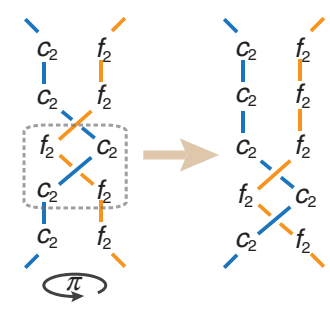

FIG. 13. (a) The configuration of the link $L_{c, f}$ with the quasiparticles associated with it in each layer. The layers are horizontal and are not drawn in this figure. In this link $L_{c, f}$, the $c$ string and the $f$ string have the linking number 1 . Their orientation is indicated by the colored arrow (blue for the $c$ string and orange for the $f$ string). (b) The operation that brings the linked part of the two strings down by one layer along the $-z$ direction is a $\pi$ rotation of the part of the link indicated by the dashed gray box. 
$\theta_{c_{2}, f_{2}}=2 \pi / p$. By similar analysis, the Berry's phase in moving the link $L_{c, f}$ one layer up toward the $z$ direction is the opposite. In the process of moving the $L_{c, f}$ around the the $d$ string [Fig. 12(g)], namely, an edge dislocation, the net effect is to move the $L_{c, f}$ by the Burgers vector, i.e., to move it downward by one layer. Therefore, we can conclude that

$$
\Omega_{L_{c, f}}^{d}=\theta_{c_{2}, f_{2}},
$$

which verifies the consistency of Eq. (55). In this discussion, we always have to keep the $d$ string fixed because it is an extrinsic defect rather than a dynamical excitation. If we can promote the $d$ strings to dynamical excitations, we can study other types of string braiding between the three types of strings $c, f$, and $d$, such as $\omega_{f, d}^{c}$ and $\omega_{d, c}^{f}$ [41]. The model that we have considered so far is not capable of capturing the dynamics of the $d$ strings, but the analysis above relating string-string braiding and link-string braiding can be used to derive general identities on the string braiding, as will be discussed in Sec. VI A.

\section{TOPOLOGICAL FIELD-THEORY DESCRIPTION}

In this section, we introduce a topological field-theory description of the layer-constructed system, which provides a phenomenological description of the particle-string braiding and string-string braiding in the presence of the edge dislocation. The field theory can be considered as a continuum limit of the discrete system of coupled layers, although a generic derivation starting from coupled layers remains an open question that we will leave for future work. In this work, we will treat the field theory as a phenomenological low-energy effective theory. We consider the following Lagrangian density:

$$
\begin{aligned}
\mathcal{L}_{\mathrm{LC}}= & \frac{Q_{I J}}{2 \pi} \epsilon^{\mu \nu \lambda \sigma} b_{\mu \nu}^{I} \partial_{\lambda} a_{\sigma}^{J}+\frac{\Theta}{8 \pi^{2}} R_{I J} \epsilon^{\mu \nu \lambda \sigma} \partial_{\mu} a_{\nu}^{I} \partial_{\lambda} a_{\sigma}^{J} \\
& +j_{\mu}^{I} a_{\mu}^{I}+\mathcal{J}_{\mu \nu}^{I} b_{\mu \nu}^{I}
\end{aligned}
$$

with the summation of repeated indices implicitly assumed. Here, $a_{\mu}^{I}$ for each $I$ is a one-form $U(1)$ gauge field that couples to the current $j_{\mu}^{I}$ of nontrivial point excitations, and $b_{\mu \nu}^{I}$ for each $I$ is a two-form $U(1)$ gauge field that couples to the two-form current $\mathcal{J}_{\mu \nu}^{I}$ of nontrivial string excitations. $\left(b_{\mu \nu}^{I}\right.$ and $\mathcal{J}_{\mu \nu}^{I}$ are antisymmetric in permutations of $\mu, \nu$.) Their gauge transformations are given by $a_{\mu}^{I} \rightarrow a_{\mu}^{I}+\partial_{\mu} \alpha^{I}$ and $b_{\mu \nu}^{I} \rightarrow b_{\mu \nu}^{I}+\partial_{\mu} \beta_{\nu}^{I}+\partial_{\nu} \beta_{\mu}^{I}$. $Q_{I J}$ and $R_{I J}$ are integervalued nonsingular matrices, and $R$ is symmetric. $\Theta$ is an "order-parameter field" that is extrinsically determined and does not have dynamics. When we consider the compactification radius of each $a_{\mu}^{I}$ as $2 \pi$, the integral $\frac{1}{8 \pi^{2}} \int d^{4} x R_{I J} \epsilon^{\mu \nu \lambda \sigma} \partial_{\mu} a_{\nu}^{I} \partial_{\lambda} a_{\sigma}^{J}$ is quantized to integer values for all closed space-time manifolds. Therefore, the partition function of the system is invariant under the transformation $\Theta \rightarrow \Theta+2 \pi$. For a manifold with a boundary, $\Theta \rightarrow \Theta+2 \pi$ will induce a boundary Chern-Simons term $\int_{\text {boundary }} d^{3} n_{\mu} \frac{1}{4 \pi} R_{I J} \epsilon^{\mu \nu \sigma \tau} a_{\nu}^{I} \partial_{\sigma} a_{\tau}^{J}$. Here, $\int d n_{\mu}$ is the boundary volume integration with $d n_{\mu}$ along the normal direction. The topological field theory (57) has the form of BF theory $[65,66]$. Similar theory has been proposed to describe fermionic and bosonic topological insulators $[26,67]$ and the Walker-Wang model $[32,33,68]$. Compared with previous works, the essential new ingredient in our theory (57) is the string-string braiding statistics, which, as will be explained in the following, is enabled by the possibility of $\Theta$ vortex loops.

We start from a system with constant $\Theta$. The equation of motion is given by

$$
\begin{aligned}
j_{\mu}^{I} & =-\frac{1}{2 \pi} Q_{J I} \epsilon^{\mu \nu \lambda \sigma} \partial_{\nu} B_{\lambda \sigma}^{J}, \\
\mathcal{J}_{\mu \nu}^{I} & =-\frac{1}{2 \pi} Q_{I J} \epsilon^{\mu \nu \lambda \sigma} \partial_{\lambda} a_{\sigma}^{J} .
\end{aligned}
$$

Therefore, the string particle carries flux of $a_{\mu}^{I}$, so that braiding the $I$ th particle $\Pi_{I}$ (whose current is given by $j_{\mu}^{I}$ ) and the $J$ th string $\Sigma_{J}$ (whose current is given by $\mathcal{J}_{\mu \nu}^{J}$ ) produces the Berry's phase

$$
\theta_{\Pi_{I}, \Sigma_{J}}=-2 \pi\left(Q^{-1}\right)_{I J}
$$

By properly choosing the matrix $Q$, the Lagrangian density $\mathcal{L}_{\mathrm{CL}}$ can capture the particle-string statistics in the layerconstructed model. For example, to capture the braiding statistics in Eq. (39), we need to take $Q=p$ (where $Q$ is a $1 \times 1$ matrix). For the case studied in Sec. IV B, we should have $Q=3$. For these two cases, $R$ 's are taken to be 0 . However, even with a nonzero matrix $R$, the string-string braiding is trivial when $\Theta$ is a constant. To obtain nontrivial string-string braiding, it is essential to consider space-timedependent $\Theta$.

Now, we can consider the case with specially varying $\Theta$. The equation of motion is given by

$$
\begin{aligned}
j_{\mu}^{I} & =-\frac{1}{2 \pi} Q_{J I} \epsilon^{\mu \nu \lambda \sigma} \partial_{\nu} B_{\lambda \sigma}^{J}-\frac{1}{4 \pi^{2}} R_{I J} \epsilon^{\mu \nu \lambda \sigma} \partial_{\nu} \Theta \partial_{\lambda} a_{\sigma}^{J}, \\
\mathcal{J}_{\mu \nu}^{I} & =-\frac{1}{2 \pi} Q_{I J} \epsilon^{\mu \nu \lambda \sigma} \partial_{\lambda} a_{\sigma}^{J} .
\end{aligned}
$$

For the case without any point particles, i.e., $j_{\mu}^{I}=0$, we have

$$
\left(Q^{-1 T} R Q^{-1}\right)_{I J} \mathcal{J}_{\mu \nu}^{J} \partial_{\nu} \Theta=\epsilon^{\mu \nu \lambda \sigma} \partial_{\nu} B_{\lambda \sigma}^{I}
$$

Since the bulk of the system is invariant under the transformation $\Theta \rightarrow \Theta+2 \pi$, we can consider a $2 \mathrm{D}$ surface in 
the system across which the the value of $\Theta$ jumps by $2 \pi$. For example, when this 2D surface is the $x y$ plane, we have

$$
\partial_{\mu} \Theta=\delta_{\mu, z} 2 \pi \delta(z)
$$

When the vortex string $\Sigma_{J}$ goes through the $z=0$ plane, the point of penetration acts as a point source for $\epsilon^{\mu \nu \lambda \sigma} \partial_{\nu} B_{\lambda \sigma}^{I}$. If we consider braiding strings $\Sigma_{I}$ and $\Sigma_{J}$, as are shown in Fig. 14(a) (with the $z=0$ plane, where $\partial_{z} \Theta=2 \pi$, is the green plane shown in the figure), the Berry's phase of this braid is

$$
\omega_{\Sigma_{I}, \Sigma_{J}}=2 \pi\left(Q^{-1 T} R Q^{-1}\right)_{I J}
$$

In this discussion, the only nontrivial effects from the $\Theta$ configuration in Eq. (62) are the braiding statistics between strings $\Sigma_{I}$ and $\Sigma_{J}$ that go through the $z=0$ only once and close at infinity. It is straightforward to see that if we consider strings of finite size, they have to cross the $z=0$ plane an even number of times and their braiding is always trivial. Therefore, the system behaves the same as the original system without the jump of $\Theta$ across $z=0$. To obtain nontrivial braiding of finite-size strings, we need to consider a 2D surface $\gamma$ with a boundary $\partial \gamma \neq \varnothing$ crossing that $\Theta$ jumps by $2 \pi$. Such a surface is a vortex loop of $\Theta$ at $\partial \gamma$. The strings that are linked with the vortex loop will have nontrivial string braiding statistics. For example, the braid statistics of the string $\Sigma_{I}$ and $\Sigma_{J}$ in Fig. 14(b) is given by Eq. (63). Compared to the result we present earlier in layer construction, we can easily identify the defected layer in Fig. 10(a) as the surface $\gamma$ and the edge dislocation as $\partial \gamma$. Since the jump of $\Theta$ is not locally observable in the bulk, we can think of the boundary $\partial \gamma$ as a line defect, which is consistent with the edge-dislocation interpretation.

For a specific layer-constructed model, we can choose proper $Q_{I J}$ and $R_{I J}$ to capture the phenomenology of the model. For example, for the example we studied in Sec. IV C, the matrix $Q_{I J}$ is determined by the statistics between point particles and string excitations in Eq. (52): (a)

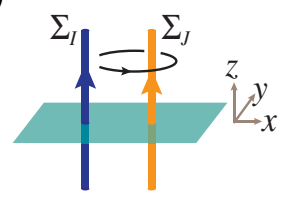

(b)

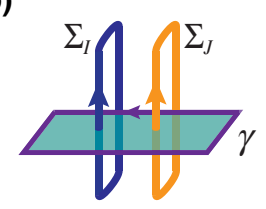

FIG. 14. (a) The configuration of two strings $\Sigma_{I}$ and $\Sigma_{J}$ that are aligned along the $z$ direction and penetrate the $z=0$ plane (green region) where $\Theta$ jumps by $2 \pi$. The strings $\Sigma_{I}$ and $\Sigma_{J}$ have nontrivial braiding. (b) The configuration of a finite open surface $\gamma$ across which $\Theta$ jumps by $2 \pi$ together with strings $\Sigma_{I}$ and $\Sigma_{J}$ that are linked with the vortex loop $\partial \gamma$ (purple border).

$$
Q=\left(\begin{array}{cc}
p / 2 & 0 \\
0 & 2
\end{array}\right)
$$

With the choice

$$
R=\left(\begin{array}{ll}
0 & 1 \\
1 & 0
\end{array}\right)
$$

we obtain the string braiding statistics

$$
2 \pi\left(Q^{-1 T} R Q^{-1}\right)_{I J}=2 \pi\left(\begin{array}{cc}
0 & 1 / p \\
1 / p & 0
\end{array}\right),
$$

which is consistent with braiding statistics $\omega_{c, f}^{d}$ between the $c$ string and the $f$ string calculated in Eq. (53) in the presence of the $d$ string.

\section{GENERAL DISCUSSION ON STRING BRAIDING}

\section{A. General identities on Abelian string braiding statistics}

In the last part of the previous section, we discuss the braiding statistics of strings that involves two dynamical string excitations and one extrinsic string defect. It is conceivable that similar string braiding can happen in the same configuration for three dynamical string excitations. As is recently discussed in Refs. [41-43], in twisted lattice gauge theories defined by nontrivial group cohomology classes, different flux strings can braid nontrivially. Reference [41] proposed an identity on braiding statistics of these flux strings. For two strings $a$ and $b$ both linked with $c$ [Fig. 15(a)], the braiding phase of $a$ and $b$ is defined as $\omega_{a, b}^{c}$. Reference [41] proved the following identity (up to $2 \pi$ times integers) for $3 \mathrm{D} Z_{N}^{k}$ gauge theories:

$$
N\left(\omega_{a, b}^{c}+\omega_{b, c}^{a}+\omega_{c, a}^{b}\right)=0 .
$$

(a)

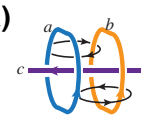

(b)

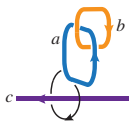

$\Omega_{L_{a, b}}^{c}$

Equivalent process to (c)

(d) (c)

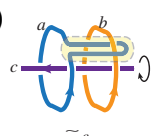

$\omega_{a, b}^{c}$

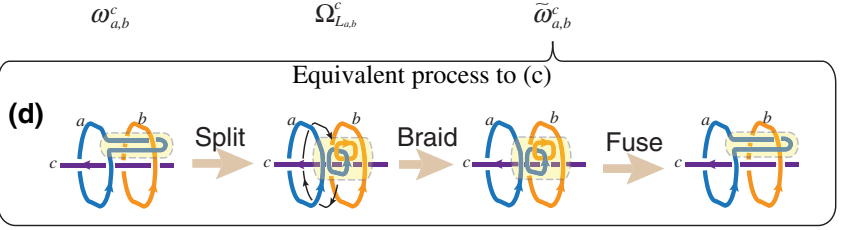

FIG. 15. Three different string braiding processes, named (a) the string-string braiding, (b) the link-string braiding, and (c) the linked braiding. As is explained in the main text, the Berry's phases associated with these three processes are identical. (d) The decomposed steps that relate processes in (c) and (b). 
In the following, we will present a more general proof of a slightly modified version of this identity for Abelian string braiding, by making use of splitting and fusion of strings.

As a general setup of our discussion, we will consider the string excitations that have the following properties: (1) The strings are Abelian in the sense that there are no nontrivial degenerate states associated with a local string configuration, (2) the strings have no pointlike excitation attached to them, (3) strings of the same type can split and fuse without inducing an additional phase factor, and (4) the strings can link with each other.

Since we have assumed that there is no nontrivial degeneracy associated with the string configuration, the Berry's phase $\omega_{a, b}^{c}$ is simply a $U(1)$ phase. This Berry's phase $\omega_{a, b}^{c}$ should be a topological invariant that does not depend on the shape of the strings in the process of braiding. In Sec. IV C, we have shown that by fusion and splitting of the same type of strings, one can topologically deform the string-string braiding process of $a$ and $b$ strings linked to $c$ [Fig. 15(a)] to a particle-string braiding process in which the "particle" $L_{a, b}$ is formed by two linked loops of types $a$ and $b$. Denoting the Berry's phase obtained by the braiding of $L_{a b}$ with string $c$ as $\Omega_{L_{a, b}}^{c}$, we obtain the equation $\omega_{a, b}^{c}=\Omega_{L_{a, b}}^{c}$. Now, we consider a different threestring configuration shown in Fig. 15(c). In this configuration, every pair of strings is mutually linked, with linking number 1 . The "braiding" process (which will be referred to as the linked braiding) in this configuration is defined to be a $2 \pi$ rotation of the two strings $a$ and $b$ about the string $c$ [see Fig. 15(c)] while keeping the relative position between $a$ and $b$ fixed. The Berry's phase associated with this process is denoted as $\tilde{\omega}_{a, b}^{c}$. Following a similar string fusion and splitting procedure as that which has been presented earlier in Fig. 15(c), we can deform the linked braiding process to the same particle-string braiding shown in Fig. 15(b). This deformation is illustrated in detail in Fig. 15(d). Therefore, we conclude that

$$
\omega_{a, b}^{c}=\Omega_{L_{a, b}}^{c}=\tilde{\omega}_{a, b}^{c} .
$$

Now, we proceed to prove a modified version of the identity (67) by proving it for $\tilde{\omega}_{a, b}^{c}$. The configuration of three mutually linked loops in Fig. 15(c) can be topologically deformed to that in Fig. 16. This configuration is symmetric upon cyclic permutation of the three strings. The "linked braiding" process defined in Fig. 15(c) can be rephrased as the operation that keeps the string $c$ fixed and rotates simultaneously the strings $a$ and $b$ about an axis, which goes through the reference point $o$ and is perpendicular to the $2 \mathrm{D}$ plane, clockwisely by $2 \pi$. This process is the linked braiding of the strings $a$ and $b$ with respect to the string $c$. Because of the cyclic symmetry between the three strings in this configuration in Fig. 15, we can also consider similar linked braiding processes

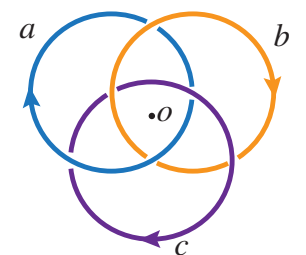

FIG. 16. The configuration of three mutually linked strings. This configuration is topologically equivalent to the configuration shown in Fig. 15(c) but is deformed to a shape with threefold rotation symmetry according to an axis perpendicular to the plane at the point $o$.

between the strings $b$ and $c$ with respect to the string $a$, and that between $c$ and $a$ with respect to $b$. If we perform these three different types of linked braiding sequentially, the net Berry's phase obtained is, by definition, $\tilde{\omega}_{a, b}^{c}+\tilde{\omega}_{b, c}^{a}+\tilde{\omega}_{c, a}^{b}$. However, one can easily see that the combined process is actually a global $4 \pi$ rotation of the whole string configuration around axis $o$, since each string is rotated by $2 \pi$ during two of the three processes and is held static during one of them. Since a $4 \pi$ rotation is a topologically trivial action in three dimensions, the total Berry's phase induced by the rotation must be trivial. It implies that the sum of three different linked braiding Berry's phases should be trivial, namely,

$$
\tilde{\omega}_{a, b}^{c}+\tilde{\omega}_{b, c}^{a}+\tilde{\omega}_{c, a}^{b}=0 .
$$

Therefore, by using Eq. (68), we prove the following identities:

$$
\begin{gathered}
\omega_{a, b}^{c}+\omega_{b, c}^{a}+\omega_{c, a}^{b}=0, \\
\Omega_{L_{a, b}}^{c}+\Omega_{L_{b, c}}^{a}+\Omega_{L_{c, a}}^{b}=0 .
\end{gathered}
$$

Equation (70) directly implies the previously proposed Eq. (67). Equation (71) constrains the braiding statistics of strings and "link particles."

Our proof here only relies on adiabatical deformations and fusion-splitting processes of strings, which are thus applicable to generic 3D topologically ordered states that involve Abelian stringlike excitations.

\section{B. Non-Abelian string braiding statistics}

The discussion above only applies to the 3D topological states with string excitations that satisfy the assumptions given in the beginning of the previous subsection. It is worthwhile to point out that there is certainly a possibility of other, more generic, stringlike excitations that violate some of these assumptions. For instance, the same string configurations can, in principle, carry non-Abelian degrees of freedom. The general framework of non-Abelian stringstring braiding is beyond the scope of this paper, but we 
would like to present one explicit example of a system with non-Abelian string-string braiding.

Consider two Weyl fermions in $3+1$ dimensions with opposite chirality, which are equivalent to a Dirac fermion. Each Weyl fermion is time-reversal invariant, and a superconducting pairing can be introduced within each Weyl fermion. The Fermi level can be either at or away from the Weyl point, which does not affect our discussion. The BCS Hamiltonian of such a superconductor can be written as

$$
\begin{aligned}
\hat{H}= & \sum_{\mathbf{k}}\left[\psi_{R \mathbf{k}}^{\dagger}\left(v_{F} \sigma \cdot \mathbf{k}-\mu\right) \psi_{R \mathbf{k}}\right. \\
& \left.+\psi_{L \mathbf{k}}^{\dagger}\left(-v_{F} \sigma \cdot \mathbf{k}-\mu\right) \psi_{L \mathbf{k}}\right] \\
& +\frac{1}{2} \sum_{\mathbf{k}}\left[\left|\Delta_{R}\right| e^{i \theta_{R}} \psi_{R-k}^{\dagger} i \sigma_{y} \psi_{R \mathbf{k}}^{\dagger}\right. \\
& \left.+\left|\Delta_{L}\right| e^{i \theta_{L}} \psi_{L-k}^{\dagger} i \sigma_{y} \psi_{L \mathbf{k}}^{\dagger}+\text { H.c. }\right],
\end{aligned}
$$

where $\psi_{R \mathbf{k}}$ and $\psi_{L \mathbf{k}}$ are two-component fermion operators, $v_{F}$ is the Fermi velocity, $\sigma$ 's are the $2 \times 2$ Pauli matrices, and $\Delta_{R}=\left|\Delta_{R}\right| e^{i \theta_{R}}$ and $\Delta_{L}=\left|\Delta_{L}\right| e^{i \theta_{L}}$ are the pairing order parameters on the Fermi surface of $\psi_{R \mathbf{k}}$ and $\psi_{L \mathbf{k}}$. Without the pairing term and with $\mu>0$, the Fermi surface of $\psi_{R \mathbf{k}}$ carries Chern number 1 while the Fermi surface of $\psi_{L \mathbf{k}}$ carries Chern number -1 . This model was studied before [69] as a minimal model of time-reversal-invariant topological superconductivity (TSC) [70-72], which describes a TSC when $\theta_{L}=0$ and $\theta_{R}=\pi$ and a trivial superconductor when $\theta_{L}=\theta_{R}=0$ [73].

As a probe to the TSC, Ref. [69] studied the chiral vortex strings in this system, which are $2 \pi$ vortex strings of only one of the pairing phases, such as $\theta_{R}$. This chiral vortex string carries a $(1+1) \mathrm{D}$ chiral Majorana fermion mode, which has an axial anomaly. For the purpose of this paper, we will focus on the zero-energy mode in the spectrum of the chiral Majorana fermion, usually named the Majorana zero mode [74]. A contractible vortex string does not carry a zero-energy mode, since the Majorana fermion has an antiperiodic boundary condition. (Without going into a detailed calculation, one can see that this absence of the zero mode must be true since a Majorana zero mode, if it exists, cannot disappear when the loop shrinks to a point and disappears. Therefore, it cannot occur in a contractible loop.) When a vortex string $v_{1}$ is linked with another chiral vortex string $v_{2}$ [see Fig. 17(a)], there will be Majorana zero modes $\gamma_{1(2)}$ on each vortex string. Each Majorana zero mode is separated from other chiral modes by a finite-size (a)

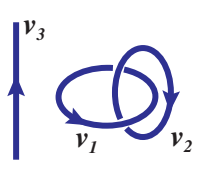

(b)

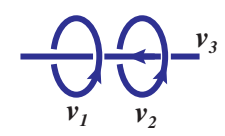

FIG. 17. (a),(b) Two different configurations of chiral vortex strings. gap that is inversely proportional to the length of the vortex strings. There are two degenerate states with the opposite fermion parity $(-1)^{F} \equiv i \gamma_{1} \gamma_{2}$ associated with the two zero modes on the two linked strings. If we braid the link between $v_{1}$ and $v_{2}$ around a third chiral vortex string $v_{3}$, the Berry's phase is equal to $(-1)^{F}$. Therefore, we see that the string braiding statistics relies on the state in the degenerate space defined by the string configuration, which is a threedimensional generalization of "fusion channels" of a nonAbelian anyon. Consider a different chiral vortex-string configuration in Fig. 17(b) with two vortex strings $v_{1}$ and $v_{2}$ both linked with $v_{3}$. In this configuration, both $v_{1}$ and $v_{2}$ carry Majorana zero modes $\gamma_{1}^{\prime}$ and $\gamma_{2}^{\prime}$ while $v_{3}$ does not have a zero mode. If we braid the vortex strings $v_{1}$ and $v_{2}$ in the way defined in Fig. 10(a), the Majorana fermion operators $\gamma_{1,2}^{\prime}$ will each obtain a -1 sign after a full braiding, due to the phase winding around the vortices. Therefore, one expects that the braiding between these two strings with zero modes is the same as the braiding of two pointlike vortices in a $(2+1) \mathrm{D} p+i p$ superconductor $[75,76]$, up to an undetermined overall phase factor. The non-Abelian Berry's phase is a unitary operator acting on the two-dimensional Hilbert space, which can be written as $e^{i\left(2 \pi i \gamma_{1}^{\prime} \gamma_{2}^{\prime} / 4\right)}$. Since the chiral vortices are generically confined with the antichiral vortices energetically [69], they have to be considered as external defects, and the Abelian phase factor of the braiding process is not well defined.

Since we can consider all the chiral vortices to be identical, we can also consider the "half-braid" between $v_{1}$ and $v_{2}$ that is similar to the string braiding defined in Fig. 10(a) but only exchanges the position of two vortex strings instead of braiding one string around the other. In this half-braid process, we can keep the sizes of all three vortex strings $v_{1}, v_{2}$, and $v_{3}$ finite. The degenerate space defined by the two zero modes $\gamma_{1,2}^{\prime}$ is separated from other excited states by a finite-size gap. Therefore, the half-braid should not change the total fermion parity $(-1)^{F}=i \gamma_{1}^{\prime} \gamma_{2}^{\prime}$ of the three-string configuration. The only possible nontrivial operation to the Majorana zero modes is

$$
\begin{aligned}
& \mathcal{U}^{\dagger} \gamma_{1}^{\prime} \mathcal{U}=\gamma_{2}^{\prime}, \\
& \mathcal{U}^{\dagger} \gamma_{2}^{\prime} \mathcal{U}=-\gamma_{1}^{\prime},
\end{aligned}
$$

where $\mathcal{U}$ is the unitary transformation induced by the halfbraid. This equation determines $\mathcal{U}=e^{2 \pi i \gamma_{1}^{\prime} \gamma_{2}^{\prime} / 8}$ up to a $U(1)$ phase.

When more generic configurations of vortex strings are considered, one can see a more qualitative difference from the Abelian case. For example, one can consider the configuration of three mutually linked chiral vortex strings shown in Fig. 16. In the Abelian case, a linked braiding procedure is defined in this configuration [Figs. 15(c) and 15(d)], which is proved to induce the same Berry's phase as the three-string braiding process in Fig. 15(a). In the case of chiral vortex strings, the configuration of three mutually 
linked strings is very different from two strings linked with the third one: When each loop is linked with two other loops, there is no Majorana zero mode on any of the three loops. Consequently, there cannot be any nontrivial nonAbelian Berry's phase in the linked braiding process. If the linked braiding and unlinked braiding [the procedures shown in Figs. 15(a) and 15(c)] are still related, the linked braiding will at most be related to a given fusion channel of the two strings in the unlinked configuration. In general, the fusion and splitting of non-Abelian strings involve the change of topological degeneracy, which is more complicated than the case of Abelian strings with "adiabatic" fusion and splitting within the same species. Fusion channels have to be defined when we consider the splitting and fusion of strings. We will leave the non-Abelian fusion of strings for future study.

One can consider a multicomponent generalization of the model (72), which has a matrix-valued pairing order parameter and allows monopolelike non-Abelian point defects in addition to the chiral vortices [36-39]. The non-Abelian statistics of monopole defects are also related to Majorana zero modes, and it is interesting to investigate the interplay of the braiding statistics of monopoles and chiral vortex strings.

There are also other similar examples in the 3D quantum double model [45] and 3D Dijkgraaf-Witten lattice theory [43] that strings can carry non-Abelian degrees of freedom and have non-Abelian braiding statistics. When we dimensionally reduce the $z$ direction to a finite compactified circle for these lattice gauge-theory systems, the strings align along the $z$ direction, which can be viewed as a quasiparticle in the 2D sense and can be assigned quantum dimensions, since the 3D lattice gauge theory, when dimensionally reduced, becomes 2D lattice gauge theory. Each quasiparticle (in the 2D theory) must have integer quantum dimension, and so must the strings (along the $z$ direction). In contrast, if we dimensionally reduce the 3D TSC to $2 \mathrm{D}$ with a $\pi$ chiral flux in the $z$-direction cycle, the chiral vortex strings (along the $z$ direction) will have quantum dimension $\sqrt{2}$ due to the Majorana zero mode. It would be interesting to find more examples of string excitations with noninteger quantum dimensions. Moreover, Refs. [41-43] mainly concern the braiding process defined in Fig. 15(a) between flux strings in the twisted Dijkgraaf-Witten lattice gauge theory. It would also be interesting to study the braiding process defined in Figs. 15(b) and 15(c) for these models.

\section{CONCLUSION AND DISCUSSION}

In this paper, we propose a general approach to obtain nontrivial 3D topological states through layer construction. Starting from stacked layers of 2D Abelian topological states, anyon condensation induces interlayer coupling and drives the system into a three-dimensional state that may have interesting bulk and/or surface topological orders. We illustrate several different possibilities with explicit examples. The first class of states is those with trivial bulk topological order and nontrivial surface twodimensional topological order. We obtain general criteria for such states and show that this state has the same topological order on surfaces with different orientation, even though it appears to be anisotropic. It implies that the layer construction indeed captures the properties of homogeneous 3D states. The second class of states is those with nontrivial bulk topological order, which means nontrivial ground-state degeneracy on a three-dimensional torus. The simplest examples realize the discrete gauge theories. More generically, the layer construction can describe a broader class of topologically ordered states that have coexisting surface and bulk topological order. One of the most interesting properties that can be realized in some layerconstructed topological states is the string-string braiding statistics, in addition to the more conventional particlestring braiding that exists in discrete gauge theories. We provide an explicit example of a state with nontrivial stringstring braiding and also discuss the relation between stringstring braiding and particle-string braiding by making use of the fusion and splitting procedures of strings. A topological field theory is proposed to describe the topological order and string braiding statistics of the layerconstructed systems. The topological field theory has the form of BF theory "twisted" by an "axionic" topological coupling of the gauge field with an axion field $\theta$. Based on the explicit examples and field theory, we further discuss the general properties of three-string braiding and give a more general proof of the string braiding identities [Eqs. (69), (70), and (71)], with Eq. (70) being a a modified version of the previously proposed identity (67) proved for $Z_{N}^{k}$ lattice gauge theories. Finally, we discuss the generalization of the 3D topological order and string-string braiding to non-Abelian systems by proposing one example system that is a superconductor with chiral vortex strings. Compared with Abelian strings, the non-Abelian strings have multiple fusion channels, a non-Abelian braiding phase, and topological degeneracy, depending on string linking.

There are a lot of interesting open questions along the direction of this work. The first question is how to write down lattice models, which realizes the coupled layers and anyon condensation. For layers of $Z_{p}$ toric code with condensation described in Fig. 6(a), the lattice model is simply the 3D toric code. This statement can be understood in the following way. Take $p=2$ as an example. Consider the cubic lattice with $Z_{2}$ degrees of freedom defined on each link. The vertex term on the vertex $v$ is given by $\mathcal{O}_{v}=$ $-\prod_{l \text { connects to } v} \sigma_{l}^{z}$ with $l$ labeling the link, and the plaquette term at plaquette $p$ is given by $\mathcal{O}_{p}=-\prod_{l \in \text { plaquette } p} \sigma_{l}^{x}$. Now, consider adding a term $h \sigma_{l}^{z}$ for all vertical links with $h$ a coupling constant. In the $h \rightarrow \infty$ limit, all the vertical links are polarized and the 3D toric code splits up into 
layers of 2D toric code. As we lower the coupling $h$, a transition that is described in Fig. 6(a) will happen and bring the system back to the topological ground state of the $3 \mathrm{D}$ toric code. Its $Z_{p}$ and twisted version can also be constructed in a similar fashion. It would be interesting to construct lattice models that capture the physics of more generic 3D states obtained through layer construction.

Another interesting question is the relation of the layerconstructed states with the Walker-Wang models. Some similarities are discussed in Sec. IV B between these two systems, and the work of Walker and Wang [32] sets a general mathematical framework of defining 3D topological states. However, string-string braiding has not been discussed in the Walker-Wang model, so it will be an interesting future direction.

If incorporated with symmetry, the layer construction for a 3D state with purely surface topological order can be used to build models for SPT states with gapped surfaces, as has been discussed in Ref. [46]. Our analysis on the side surface can serve as a Hamiltonian formalism for these topologically ordered surfaces of 3D SPTs, which allows us to study more carefully the symmetry-breaking transition on the surface. In particular, one can use this formalism to study the symmetry-broken surface states and the symmetry-breaking surface domain wall of the $e T m T$ phase of the 3D bosonic topological insulator. Here, we have adopted the notation $e T m T$ from Ref. [46]. " $e T m T$ " is the label for the time-reversal-invariant bosonic topological insulator that admits a symmetry-preserving gapped surface with $Z_{2}$ toric code surface topological order in which both the electric and the magnetic particles transform as a Kramers doublet under time reversal. The reason to study this case, in particular, is that the time-reversal symmetrybreaking surface domain walls on the surface of the $e T m T$ phase do not carry nontrivial gapless degrees of freedom. Yet, we still cannot proliferate them to restore the symmetry without introducing surface topological order. It would be interesting to study the properties of domain walls using the Hamiltonian formalism to understand this phenomenon.

Another future direction is a more systematic understanding of the 3D topological order. For a 3D gapped state with purely surface topological order and trivial bulk topological order, we have obtained some general criteria on when it occurs and what is the surface topological order (Sec. III B). However, for more generic 3D states with bulk and/or surface topological order, we have only done a caseby-case study of the topological order for each given set of condensed anyons. More specifically, what is needed is a general formula that determines the topological properties of the 3D state (such as the $Q$ and $R$ matrices in the fieldtheory approach) from the "microscopic" data, i.e., the layer $K$ matrix before the anyon condensation, and the condensed anyons given by $p_{i}$ and $q_{i}$ vectors. It is also interesting to consider anyon condensation and layer construction in non-Abelian layers, using the technique developed in the literature $[55,77,78]$. A specific question will be how to obtain the topological superconductor state with a chiral vortex from layer construction.

Moreover, we would like to make some more general comments on the string braiding statistics in 3D topologically ordered states. In Sec. VI, we have shown that, for Abelian strings with trivial fusion and splitting within the same species, the string braiding statistics can be defined in various different ways with configurations of different linking numbers, which yield exactly the same Berry's phase. It implies that linked string configuration (Fig. 16) might be the objects that play a central role in 3D topological order in general. For example, by applying our analysis on the identification between three-string braiding [Fig. 15(a)] and the link-string braiding [Fig. 15(b)] to the Dijkgraaf-Witten lattice $Z_{N}^{k}$ or $Z_{N_{1}} \times Z_{N_{2}} \times Z_{N_{3}} \times Z_{N_{4}}$ gauge theories studied in Refs. [41-43], we immediately conclude that the link between two flux strings is, in fact, charged under the gauge group. Further, our example of a chiral vortex in TSC and the result in Refs. [43,45] show that the link of flux strings or themselves can even carry nonAbelian degrees of freedom. It would be very interesting to construct the mathematical framework that captures the braiding, linking, and fusion of the strings and study the various operations to the linked string configurations discussed in Sec. VI in generality. Also, another interesting direction is to reproduce the Dijkgraaf-Witten lattice gaugetheory model using the layer construction. With a different perspective of these lattice gauge theories from the layerconstructed models, one might be able to understand more about the properties of string-string braiding statistics.

In terms of topological order in more than $2+1$ dimensions, Ref. [64] recently proposed a general theory for topological order in any dimension. In Sec. IV B, we discuss the relation between the anomalous surface theory in the presence of bulk topological order in the layer construction and anomaly for gap theories proposed in Ref. [64]. It would be interesting to study further the connection of this proposal for general topological order to the layer-constructed models and to the general properties of string statistics in 3D.

At a final comment, we would like to discuss the experimental implication of our layer construction. In our setting, the starting point of our construction is the stacked layers of 2D (nonchiral) topological states. A similar setting was considered in Ref. [79] in which a stacked layer of Laughlin states is coupled and gapless photon modes emerge. To realize these layer-constructed systems in experiment, the crystal graphite should be a good candidate material. The reason is that graphite is naturally stacked layers of graphene, which is capable of hosting many nontrivial 2D topological states [80-85]. It was proposed in Ref. [86] that under a magnetic field, the crystal graphite can exhibit the 3D quantum Hall effect. When we move toward the strongly correlated regime of 
graphite under a magnetic field, this setting can, in principle, allow us to realize the system proposed in Ref. [79]. For our layer-constructed 3D topological state, since each layer in this construction is nonchiral, we can start directly with stacked layers of graphene, namely, graphite crystal, without the external magnetic field. It is possible that, in the strongly correlated regime, certain interactions can stabilize the phases proposed using the layer construction. The exact form of such interactions needs to be worked out more carefully in future works.

\section{ACKNOWLEDGMENTS}

We would like to thank Daniel Arovas, Maissam Barkeshli, Ling-Yan Hung, Senthil Todadri, Chong Wang, Zhenghan Wang, and Xiao-Gang Wen for helpful discussions. This work is supported by the Bright Oceans Corporation (C.-M. J.), the Alfred P. Sloan Foundation (X.-L. Q.) and the David and Lucile Packard Foundation (X.-L. Q.).

[1] X.-G. Wen, Quantum Field Theory of Many-Body Systems (Oxford University Press, Oxford, England, 2004).

[2] K. v. Klitzing, G. Dorda, and M. Pepper, New Method for High-Accuracy Determination of the Fine-Structure Constant Based on Quantized Hall Resistance, Phys. Rev. Lett. 45, 494 (1980).

[3] D. C. Tsui, H. L. Stormer, and A.C. Gossard, TwoDimensional Magnetotransport in the Extreme Quantum Limit, Phys. Rev. Lett. 48, 1559 (1982).

[4] R. B. Laughlin, Anomalous Quantum Hall Effect: An Incompressible Quantum Fluid with Fractionally Charged Excitations, Phys. Rev. Lett. 50, 1395 (1983).

[5] G. Moore and N. Read, Nonabelions in the Fractional Quantum Hall Effect, Nucl. Phys. B360, 362 (1991).

[6] X.-G. Wen and Z. Wang, Classification of Symmetric Polynomials of Infinite Variables: Construction of Abelian and Non-Abelian Quantum Hall States, Phys. Rev. B 77, 235108 (2008).

[7] B. Andrei Bernevig and F. D. M. Haldane, Model Fractional Quantum Hall States and Jack Polynomials, Phys. Rev. Lett. 100, 246802 (2008).

[8] X. G. Wen, Theory of the Edge States in Fractional Quantum Hall Effects, Int. J. Mod. Phys. B 06, 1711 (1992).

[9] S. C. Zhang, The Chern-Simons-Landau-Ginzburg Theory of the Fractional Quantum Hall Effect, Int. J. Mod. Phys. B 06, 25 (1992).

[10] A. Yu. Kitaev, Fault-Tolerant Quantum Computation by Anyons, Ann. Phys. (Amsterdam) 303, 2 (2003).

[11] X.-G. Wen, Quantum Orders in an Exact Soluble Model, Phys. Rev. Lett. 90, 016803 (2003).

[12] A. Kitaev, Anyons in an Exactly Solved Model and Beyond, Ann. Phys. (Amsterdam) 321, 2 (2006).

[13] M. A. Levin and X.-G. Wen, String-Net Condensation: A Physical Mechanism for Topological Phases, Phys. Rev. B 71, 045110 (2005).

[14] F. Verstraete, M. M. Wolf, D. Perez-Garcia, and J. I. Cirac, Criticality, the Area Law, and the Computational Power of
Projected Entangled Pair States, Phys. Rev. Lett. 96, 220601 (2006).

[15] Z.-C. Gu, M. Levin, B. Swingle, and X.-G. Wen, TensorProduct Representations for String-Net Condensed States, Phys. Rev. B 79, 085118 (2009).

[16] M. P. Zaletel and R. S. K. Mong, Exact Matrix Product States for Quantum Hall Wave Functions, Phys. Rev. B 86, 245305 (2012).

[17] B. Estienne, N. Regnault, and B. A. Bernevig, Fractional Quantum Hall Matrix Product States for Interacting Conformal Field Theories, arXiv:1311.2936.

[18] T. B. Wahl, H.-H. Tu, N. Schuch, and J. I. Cirac, Projected Entangled-Pair States Can Describe Chiral Topological States, Phys. Rev. Lett. 111, 236805 (2013).

[19] J. Dubail and N. Read, Tensor Network Trial States for Chiral Topological Phases in Two Dimensions, arXiv:1307.7726.

[20] Z. Wang, Topological Quantum Computation (American Mathematics Society, Providence, RI, 2010).

[21] C. Nayak, S. H. Simon, A. Stern, M. Freedman, and S. Das Sarma, Non-Abelian Anyons and Topological Quantum Computation, Rev. Mod. Phys. 80, 1083 (2008).

[22] M. Z. Hasan and C. L. Kane, Colloquium: Topological Insulators, Rev. Mod. Phys. 82, 3045 (2010).

[23] X.-L. Qi and S.-C. Zhang, Topological Insulators and Superconductors, Rev. Mod. Phys. 83, 1057 (2011).

[24] X. Chen, Z.-X. Liu, and X.-G. Wen, Two-Dimensional Symmetry-Protected Topological Orders and Their Protected Gapless Edge Excitations, Phys. Rev. B 84, 235141 (2011).

[25] L.-Y. Hung and X.-G. Wen, Quantized Topological Terms in Weak-Coupling Gauge Theories with a Global Symmetry and Their Connection to Symmetry-Enriched Topological Phases, Phys. Rev. B 87, 165107 (2013).

[26] A. Vishwanath and T. Senthil, Physics of ThreeDimensional Bosonic Topological Insulators: SurfaceDeconfined Criticality and Quantized Magnetoelectric Effect, Phys. Rev. X 3, 011016 (2013).

[27] T. Senthil, Symmetry Protected Topological Phases of Quantum Matter, arXiv:1405.4015.

[28] B. A. Bernevig and S. C. Zhang, Quantum Spin Hall Effect, Phys. Rev. Lett. 96, 106802 (2006).

[29] M. Levin and A. Stern, Fractional Topological Insulators, Phys. Rev. Lett. 103, 196803 (2009).

[30] J. Maciejko, X.-L. Qi, A. Karch, and S.-C. Zhang, Fractional Topological Insulators in Three Dimensions, Phys. Rev. Lett. 105, 246809 (2010).

[31] B. Swingle, M. Barkeshli, J. McGreevy, and T. Senthil, Correlated Topological Insulators and the Fractional Magnetoelectric Effect, Phys. Rev. B 83, 195139 (2011).

[32] K. Walker and Z. Wang, $(3+1)-T Q F T s$ and Topological Insulators, Frontiers Phys. 7, 150 (2012).

[33] C. W. von Keyserlingk, F. J. Burnell, and S. H. Simon, Three-Dimensional Topological Lattice Models with Surface Anyons, Phys. Rev. B 87, 045107 (2013).

[34] S. Doplicher, R. Haag, and J. E. Roberts, Local Observables and Particle Statistics. I, Commun. Math. Phys. 23, 199 (1971).

[35] S. Doplicher, R. Haag, and J. E. Roberts, Local Observables and Particle Statistics. II, Commun. Math. Phys. 35, 49 (1974). 
[36] J. C. Y. Teo and C. L. Kane, Majorana Fermions and NonAbelian Statistics in Three Dimensions, Phys. Rev. Lett. 104, 046401 (2010).

[37] Y. Ran, P. Hosur, and A. Vishwanath, Fermionic Hopf Solitons and Berry Phase in Topological Surface Superconductors, Phys. Rev. B 84, 184501 (2011).

[38] M. Freedman, M. B. Hastings, C. Nayak, X.-L. Qi, K. Walker, and Z. Wang, Projective Ribbon Permutation Statistics: A Remnant of Non-Abelian Braiding in Higher Dimensions, Phys. Rev. B 83, 115132 (2011).

[39] M. Freedman, M. B. Hastings, C. Nayak, and X.-L. Qi, Weakly Coupled Non-Abelian Anyons in Three Dimensions, Phys. Rev. B 84, 245119 (2011).

[40] R. Dijkgraaf and E. Witten, Topological Gauge Theories and Group Cohomology, Commun. Math. Phys. 129, 393 (1990).

[41] C. Wang and M. Levin, Braiding Statistics of Loop Excitations in Three Dimensions, Phys. Rev. Lett. 113, 080403 (2014).

[42] S. Jiang, A. Mesaros, and Y. Ran, Generalized Modular Transformations in 3 $+1 \mathrm{D}$ Topologically Ordered Phases and Triple Linking Invariant of Loop Braiding, Phys. Rev. X 4, 031048 (2014).

[43] J. Wang and X.-G. Wen, Non-Abelian String and Particle Braiding in Topological Order-Modular $\operatorname{SL}(3, Z)$ Representation and 3+1D Twisted Gauge Theory, arXiv:1404.7854.

[44] S. Beigi, P. W. Shor, and D. Whalen, The Quantum Double Model with Boundary: Condensations and Symmetries, Commun. Math. Phys. 306, 663 (2011).

[45] H. Moradi and X.-G. Wen, Universal Topological Data for Gapped Quantum Liquids in Three Dimensions and Fusion Algebra for Non-Abelian String Excitations, arXiv:1404.4618.

[46] C. Wang and T. Senthil, Boson Topological Insulators: A Window into Highly Entangled Quantum Phases, Phys. Rev. B 87, 235122 (2013).

[47] S. L. Sondhi and K. Yang, Sliding Phases via Magnetic Fields, Phys. Rev. B 63, 054430 (2001).

[48] C. L. Kane, R. Mukhopadhyay, and T. C. Lubensky, Fractional Quantum Hall Effect in an Array of Quantum Wires, Phys. Rev. Lett. 88, 036401 (2002).

[49] J. C. Y. Teo and C. L. Kane, From Luttinger Liquid to Non-Abelian Quantum Hall States, Phys. Rev. B 89, 085101 (2014).

[50] T. Neupert, C. Chamon, C. Mudry, and R. Thomale, Wire Deconstructionism and Classification of Topological Phases, arXiv:1403.0953.

[51] F. J. Burnell, X. Chen, L. Fidkowski, and A. Vishwanath, Exactly Soluble Model of a 3D Symmetry Protected Topological Phase of Bosons with Surface Topological Order, arXiv:1302.7072.

[52] M. Barkeshli, C.-M. Jian, and X.-L. Qi, Classification of Topological Defects in Abelian Topological States, Phys. Rev. B 88, 241103 (2013).

[53] M. Barkeshli, C.-M. Jian, and X.-L. Qi, Theory of Defects in Abelian Topological States, Phys. Rev. B 88, 235103 (2013).

[54] M. Levin, Protected Edge Modes without Symmetry, Phys. Rev. X 3, 021009 (2013).
[55] L. Kong, Anyon Condensation and Tensor Categories, Nucl. Phys. B886, 436 (2014).

[56] X.-G. Wen and A. Zee, Classification of Abelian Quantum Hall States and Matrix Formulation of Topological Fluids, Phys. Rev. B 46, 2290 (1992).

[57] More rigorously, this correspondence only applies when one is allowed to topologically deform the system by adding topologically trivial blocks to the $K$ matrix. More details were discussed in Refs. [52-54].

[58] N. Read and S. Sachdev, Large-n Expansion for Frustrated Quantum Antiferromagnets, Phys. Rev. Lett. 66, 1773 (1991).

[59] Z. Wang, Topological Quantum Computation (American Mathematical Society, Providence, RI, 2010).

[60] X. G. Wen, Chiral Luttinger Liquid and the Edge Excitations in the Fractional Quantum Hall States, Phys. Rev. B 41, 12838 (1990).

[61] A. Kitaev and L. Kong, Models for Gapped Boundaries and Domain Walls, Commun. Math. Phys. 313, 351 (2012).

[62] M. Barkeshli, C.-M. Jian, and X.-L. Qi, Twist Defects and Projective Non-Abelian Braiding Statistics, Phys. Rev. B 87, 045130 (2013).

[63] A. Mesaros, Y. Baek Kim, and Y. Ran, Changing Topology by Topological Defects in Three-Dimensional Topologically Ordered Phases, Phys. Rev. B 88, 035141 (2013).

[64] L. Kong and X.-G. Wen, Braided Fusion Categories, Gravitational Anomalies, and the Mathematical Framework for Topological Orders in Any Dimensions, arXiv:1405.5858.

[65] D. Birmingham, M. Blau, M. Rakowski, and G. Thompson, Topological Field Theory, Phys. Rep. 209, 129 (1991).

[66] T. H. Hansson, V. Oganesyan, and S. L. Sondhi, Superconductors Are Topologically Ordered, Ann. Phys. (Amsterdam) 313, 497 (2004).

[67] G. Young Cho and J.E. Moore, Topological BF Field Theory Description of Topological Insulators, Ann. Phys. (Amsterdam) 326, 1515 (2011).

[68] C. W. von Keyserlingk and F. J. Burnell, Walker-Wang Models and Axion Electrodynamics, arXiv:1405.2988.

[69] X.-L. Qi, E. Witten, and S.-C. Zhang, Axion Topological Field Theory of Topological Superconductors, Phys. Rev. B 87, 134519 (2013).

[70] X.-L. Qi, T. L. Hughes, S. Raghu, and S.-C. Zhang, Time-Reversal-Invariant Topological Superconductors and Superfluids in Two and Three Dimensions, Phys. Rev. Lett. 102, 187001 (2009).

[71] R. Roy, Topological Superfluids with Time Reversal Symmetry, arXiv:0803.2868.

[72] A. P. Schnyder, S. Ryu, A. Furusaki, and A. W. W. Ludwig, Classification of Topological Insulators and Superconductors in Three Spatial Dimensions, Phys. Rev. B 78, 195125 (2008).

[73] X.-L. Qi, T. L. Hughes, and S.-C. Zhang, Topological Invariants for the Fermi Surface of a Time-ReversalInvariant Superconductor, Phys. Rev. B 81, 134508 (2010).

[74] A. Y. Kitaev, Unpaired Majorana Fermions in Quantum Wires, Phys. Usp. 44, 131 (2001).

[75] N. Read and D. Green, Paired States of Fermions in Two Dimensions with Breaking of Parity and Time-Reversal 
Symmetries and the Fractional Quantum Hall Effect, Phys. Rev. B 61, 10267 (2000).

[76] D. A. Ivanov, Non-Abelian Statistics of Half-Quantum Vortices in p-Wave Superconductors, Phys. Rev. Lett. 86, 268 (2001).

[77] F. A. Bais and J. K. Slingerland, Condensate-Induced Transitions between Topologically Ordered Phases, Phys. Rev. B 79, 045316 (2009).

[78] I. S. Eliëns, J. C. Romers, and F. A. Bais, Diagrammatics for Bose Condensation in Anyon Theories, arXiv:1310.6001.

[79] M. Levin and M. P. A. Fisher, Gapless Layered ThreeDimensional Fractional Quantum Hall States, Phys. Rev. B 79, 235315 (2009).

[80] K. S. Novoselov, A. K. Geim, S. V. Morozov, D. Jiang, M. I. Katsnelson, I. V. Grigorieva, S. V. Dubonos, and A. A. Firsov, Two-Dimensional Gas of Massless Dirac Fermions in Graphene, Nature (London) 438, 197 (2005).
[81] Y. Zhang, Y.-W. Tan, H. L. Stormer, and P. Kim, Experimental Observation of the Quantum Hall Effect and Berry's Phase in Graphene, Nature (London) 438, 201 (2005).

[82] C. Tőke, P. E. Lammert, V. H. Crespi, and J. K. Jain, Fractional Quantum Hall Effect in Graphene, Phys. Rev. B 74, 235417 (2006).

[83] K. Yang, S. Das Sarma, and A. H. MacDonald, Collective Modes and Skyrmion Excitations in Graphene SU(4) Quantum Hall Ferromagnets, Phys. Rev. B 74, 075423 (2006).

[84] K. Nomura and A. H. MacDonald, Quantum Hall Ferromagnetism in Graphene, Phys. Rev. Lett. 96, 256602 (2006).

[85] V. M. Apalkov and T. Chakraborty, Fractional Quantum Hall States of Dirac Electrons in Graphene, Phys. Rev. Lett. 97, 126801 (2006).

[86] B. A. Bernevig, T. L. Hughes, S. Raghu, and D. P. Arovas, Theory of the Three-Dimensional Quantum Hall Effect in Graphite, Phys. Rev. Lett. 99, 146804 (2007). 